\title{
LA FAMILIA ASTERACEAE EN LA FLORA DEL BAJÍO Y DE REGIONES ADYACENTES
}

\author{
José Luis VilLaSeÑoR ${ }^{1}$ y ENRIQUe Ortiz \\ Instituto de Biología, Universidad Nacional Autónoma de México, \\ Departamento de Botánica. Apdo. postal 70-233, 04510 México, D.F., México. \\ 1'Autor para la correspondencia: vrios@ibiologia.unam.mx
}

\section{RESUMEN}

Con base en ejemplares de herbario recolectados, identificados y georreferenciados provenientes del territorio considerado por el proyecto Flora del Bajío y de regiones adyacentes, se analiza la riqueza de Asteraceae conocida en dicha región. Utilizando una división de la región en cuadros de $0.5^{\circ} \times 0.5^{\circ}$, el objetivo fue identificar los sitios de alta diversidad para la familia. La región tiene documentadas, con los ejemplares de herbario revisados, 681 especies de Asteraceae, distribuidas en 182 géneros; aquellos con mayor número de especies son Ageratina (39), Stevia (31), Verbesina (29) y Acourtia (27), mientras que 82 registran una sola. En promedio se encontró una proporción de 3.7 especies por género. Querétaro es la entidad federativa que más especies registró (504), seguida de Michoacán (490) y Guanajuato (389). Análisis estadísticos proyectan la existencia de 773 especies para la región de estudio, de las cuales se han registrado 681 ( $88 \%$ de las especies esperadas; de ese porcentaje faltante, al menos 66 (67\%) han sido reportadas de alguna parte del Bajío y regiones adyacentes, pero hasta la fecha no se han observado ejemplares de herbario que respalden su presencia. Se evalúa la riqueza por cuadro y se proponen algunos considerados importantes para llevar a cabo futuras estrategias de conservación con base en los patrones de riqueza encontrados.

Palabras clave: Asteraceae, Bajío, Guanajuato, México, Michoacán, Querétaro, riqueza de especies.

\section{ABSTRACT}

Based on herbarium specimens identified and georeferenced, collected in the territory considered for the Flora of the Bajío and adjacent regions project, the family Asteraceae 
richness known for the region is analyzed. The area was divided in grid squares of $0.5^{\circ} \times 0.5^{\circ}$ size to identify areas with high diversity for the family. Currently the family has 681 species in the Bajío region, distributed in 182 genera; those with the largest number of species are Ageratina (39), Stevia (31), Verbesina (29), and Acourtia (27), whereas 82 genera have one species. On average there are 3.7 species per genus. Querétaro is the state with most species (504), followed by Michoacán (490) and Guanajuato (389). Statistical analyses project 773 species for the studied region of which 681 (88\%) have been recorded; from them, at least $66(67 \%)$ have been cited somewhere in the region, but no studied herbarium specimen until now has corroborated their presence. The floristic richness of Asteraceae is evaluated by square grids and several of them are proposed as important in future conservation strategies.

Key words: Asteraceae, Bajío, Guanajuato, México, Michoacán, Querétaro, species richness.

\section{INTRODUCCIÓN}

Es bien conocido que el Neotrópico es la región biogeográfica con la mayor biodiversidad del planeta (Myers et al., 2000). En particular México es considerado un sitio de concentración de diversidad y de endemicidad, comparado con muchas otras entidades políticas en el mundo (Mittermeier, 1988; Villaseñor, 2003). Los inventarios florísticos concluidos o en proceso están revelando regiones particularmente sobresalientes en diversidad vegetal; investigaciones en curso sugieren que la riqueza mínima promedio por unidad de área (utilizando una división del país en cuadros de $1^{\circ} \times 1^{\circ}$ ) es de 750 especies (García-Cruz et al., en preparación), aunque hay cuadros que tienen ya reportados valores superiores a las 3000 especies. Es todavía difícil, sin embargo, para muchos sitios del país definir con mayor precisión la riqueza florística existente, debido al pobre esfuerzo de recolecta realizado; otras regiones ya tienen tan degradado el ambiente que será difícil saber qué diversidad contenían, pues en muchas de ellas ya solamente se registran especies asociadas al disturbio antropogénico.

La región del Bajío está ubicada en la porción central del país y comprende los estados de Guanajuato y Querétaro y la porción noroeste de Michoacán (Fig. 1). Para muchas personas es una zona con un impacto humano negativo importante, que ha generado una disminución considerable de la cobertura vegetal original. Una evaluación del mapa de vegetación potencial del Instituto Nacional de Estadística y Geografía (Serie III, Anónimo, 2005), modificado para considerar en conjunto la 


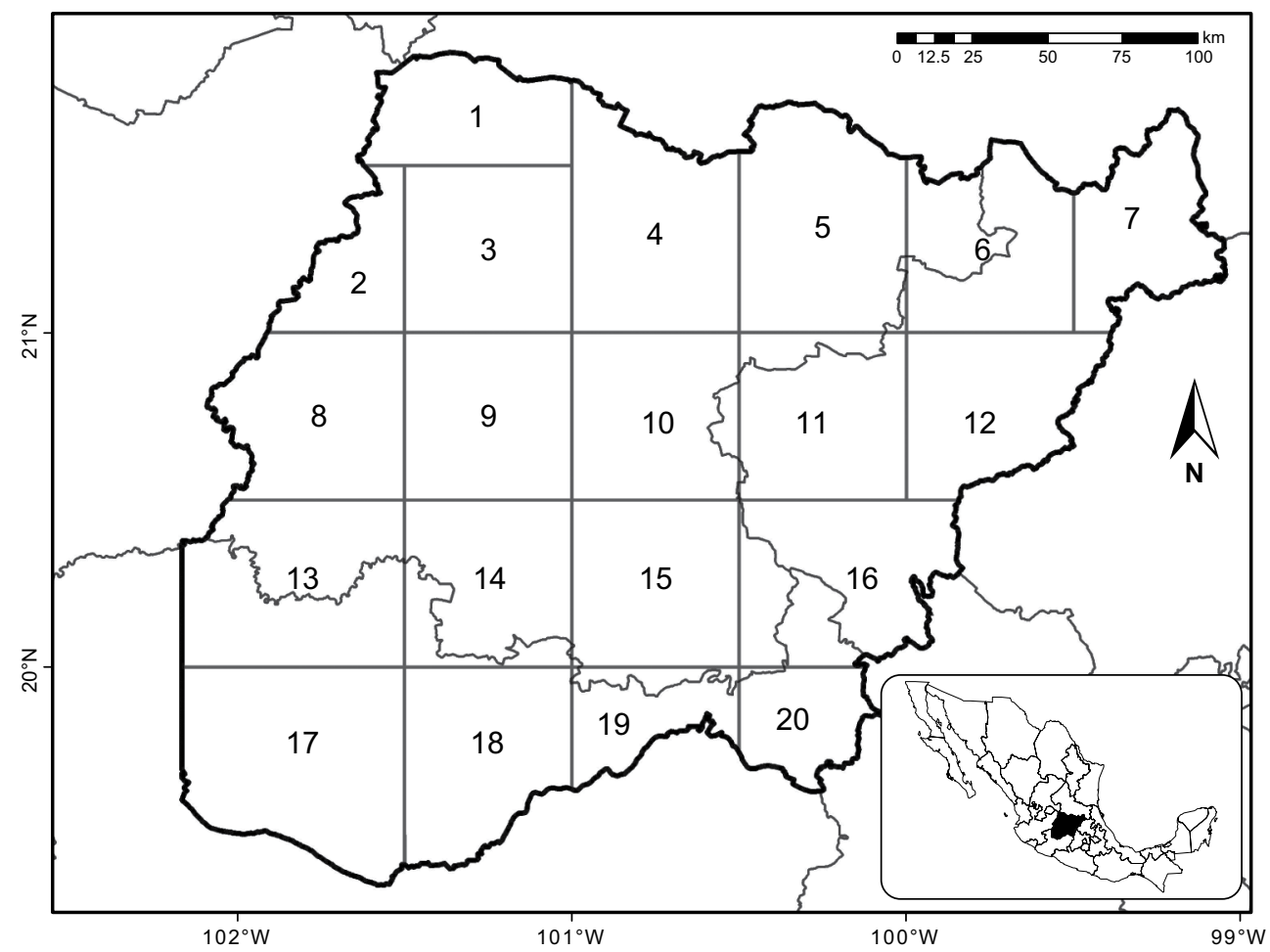

Fig. 1. Zona de estudio considerada por el proyecto Flora del Bajío y de regiones adyacentes. El territorio fue dividido en cuadros de $0.5^{\circ} \times 0.5^{\circ}$, algunos de ellos con relativamente poca superficie en la zona de estudio fusionados con sus cuadros vecinos.

porción con cobertura vegetal conservada (sin discriminar los diferentes tipos de vegetación) y sin vegetación aparente señala que la región del Bajío conserva nada más $44.4 \%$ de su cobertura original, pues $56.4 \%$ de su territorio ya es área ocupada por zonas agrícolas, urbanas o industriales. Debido a la importante influencia humana en la transformación de las comunidades vegetales originales, por muchos años se ha considerado que el Bajío mexicano es una zona con relativa pobreza de especies, visión que el proyecto "Flora del Bajío y de regiones adyacentes" ha venido a desmentir. Los resultados del mismo demuestran que la región alberga una importante proporción de la diversidad vegetal existente en el país.

La familia Asteraceae (o Compositae) es un substancial componente florístico, tanto de regiones con impacto antropocéntrico como de comunidades vegetales que todavía conservan su flora autóctona. De hecho ha sido utilizada como un 
substituto o representante de la diversidad vegetal total; su correlación positiva y significativa con los demás miembros vegetales en inventarios florísticos de diferentes sitios del territorio nacional ha permitido utilizarla como modelo para estimar valores de riqueza total (Rzedowski, 1991; Villaseñor et al., 2007). Esta relación se mantiene en la región del Bajío y de las áreas adyacentes, pues con los inventarios locales que se han registrado en la zona hasta la fecha (Cuadro 1), se observa una importante correlación positiva entre las especies de Asteraceae y el total registrado en dichos inventarios (Fig. 2). La relación entre ambos grupos indica que en promedio, por cada especie de Asteraceae existen 6.3 de otras familias de plantas vasculares $($ Mínimo $=3.8$, Máximo $=12.6$, Desviación estándar $=2.4)$, un número menor a las 10 estimadas en promedio por Rzedowski (1991) a nivel nacional.

Cuadro 1. Inventarios florísticos llevados a cabo en el territorio que comprende el proyecto Flora del Bajío y de regiones adyacentes. El número en la columna Flora refiere a un código de archivo de la información del inventario respectivo en una base de datos.

\begin{tabular}{ccccl}
\hline Flora & Estado & $\begin{array}{c}\text { Total de } \\
\text { especies }\end{array}$ & $\begin{array}{c}\text { Asteraceae } \\
\text { especies }\end{array}$ & Referencia \\
\hline 161a & Guanajuato & 197 & 36 & Quero, 1984 \\
161b & Guanajuato & 440 & 95 & Martínez-Cruz y Téllez-Valdés, 2004 \\
176a & Guanajuato & 239 & 58 & Meagher, 1994 \\
176b & Guanajuato & 271 & 46 & Gómez y Sánchez, 1999 \\
191a & Michoacán & 975 & 198 & Medina y Rodríguez, 1993 \\
191b & Michoacán & 706 & 149 & Pérez-Calix, 1996 \\
191c & Michoacán & 768 & 159 & Lot y Novelo, 1988 \\
191d & Michoacán & 1313 & 270 & Espinosa y Rodríguez-Jiménez, 1995, 1996; \\
& & & & Rodríguez-Jiménez y Espinosa, 1995, \\
& & & & 1996a; 1996b \\
192a & Michoacán & 356 & 60 & Martínez et al., 1987 \\
192b & Michoacán & 197 & 137 & Espejo et al., 1992; Cornejo-Tenorio et al., \\
& & & & 2003 \\
163a & Querétaro & 660 & 107 & Zamudio, 1984 \\
163b & Querétaro & 710 & 70 & Cartujano et al., 2002 \\
163c & Querétaro & 1361 & 156 & Arreguín-Sánchez y Fernández, 2004 \\
176c & Querétaro & 164 & 13 & Baltazar et al., 2004 \\
176d & Querétaro & 229 & 28 & Cabrera y Gómez, 2005 \\
177a & Querétaro & 281 & 51 & Fernández y Colmenero, 1997 \\
\hline & & & &
\end{tabular}




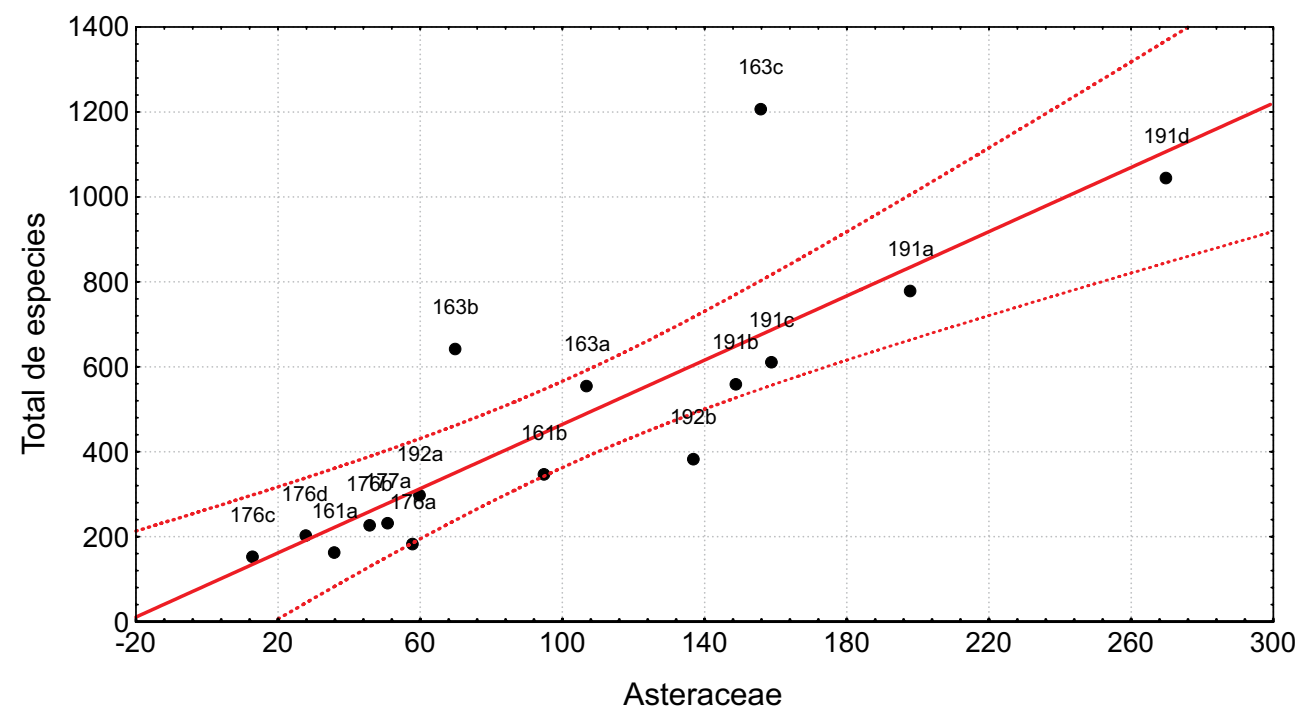

Fig. 2. Correlación entre la riqueza total y la riqueza de Asteraceae en inventarios florísticos realizados en el territorio que comprende el proyecto Flora del Bajío y de regiones adyacentes $(\mathrm{N}=16, \mathrm{r}=0.834, \mathrm{P}<0.005)$. La simbología de los puntos se explica en el Cuadro 1.

La correlación positiva $(\mathrm{r}=0.834, \mathrm{P}<0.005)$ encontrada sugiere que las Asteraceae pueden ser un buen substituto de la riqueza florística del área de estudio. Por lo tanto, utilizando como modelo a la familia, este trabajo intenta en primer lugar evaluar los patrones de su diversidad en la región, que con mucha probabilidad reflejan los patrones de toda la riqueza florística. Una vez determinados estos últimos, otros objetivos fueron identificar zonas de alta diversidad, así como partes donde es necesario llevar a cabo más trabajo de campo para lograr una mejor representación de la flora regional. Se proponen finalmente algunas recomendaciones que pueden ayudar en la conservación de su fuertemente amenazada riqueza florística.

\section{MATERIALES Y MÉTODOS}

La región considerada por el proyecto florístico "Flora del Bajío y de regiones adyacentes" fue dividida en 32 cuadros de $0.5^{\circ}$ x $0.5^{\circ}$ utilizando los paralelos y meridianos como líneas de referencia (Fig. 1). Algunos cuadros con poca superficie incluida en la zona de estudio se fusionaron con los aledaños, tratando de homegenizar lo mejor posible tanto el tamaño del área como la información registrada en ellos. 
El tamaño del cuadro seleccionado es el que mejor resuelve el compromiso entre la información recopilada y su distribución a lo largo del área de estudio; su extensión es semejante al utilizado por otros autores que han llevado a cabo trabajos similares y al parecer es una escala adecuada para evaluar la información biológica (no nada más vegetal) disponible en la actualidad (Arita et al., 1997; Ibarra-Manríquez et al., 2002; Balleza et al., 2005). De esta manera, la región de estudio fue reagrupada en 20 cuadros (Fig. 1) y para cada uno de ellos se registró la presencia de las especies de Asteraceae identificadas con el material de herbario revisado.

La lista de especies de Asteraceae presentada en este trabajo proviene por una parte de la revisión crítica de la literatura disponible, especialmente de los fascículos que presentan los resultados para algunas tribus en la región (García y Koch, 1995; Calderón, 1997; Villarreal et al., 2006; Rzedowski y Calderón, 1997; Rzedowski y Calderón, 2008; Rzedowski et al. 2011). Otro conjunto de información proviene de la revisión de más de 8200 ejemplares de herbario (sin contar duplicados), depositados en diversos herbarios tanto de México como del extranjero; gran parte de este material ya se encuentra disponible en bases de datos en línea, tanto en la Red Mundial de Información Biótica (REMIB) de la Comisión Nacional para el Conocimiento y Uso de la Biodiversidad (Conabio), como en la Unidad de Bioinformática (Unibio) del Instituto de Biología de la Universidad Nacional Autónoma de México.

Utilizando los datos geográficos y temporales de los registros de recolecta que documentan la riqueza de Asteraceae en la región, se generó una curva de acumulación de especies (Gotelli y Colwell, 2001). Para optimizar el modelo, cada cuadro de $0.5^{\circ} \times 0.5^{\circ}$ se dividió en cuatro espacios, con el fin de utilizar una escala más fina (cuadros de $0.25^{\circ} \times 0.25^{\circ}$ ). La asíntota de dicha curva de acumulación (Fig. 3) teóricamente se relaciona con el número de especies que deberíamos encontrar en la zona de estudio (Jiménez-Valverde y Hortal, 2003). Para generar la curva de acumulación y calcular su asíntota se utilizó el programa EstimateS (Colwell, 2009), con ello es posible evaluar el nivel de completitud de la flora bajo estudio.

Las semejanzas florísticas entre los cuadros fueron exploradas utilizando el coeficiente de similitud de Sorensen-Dice. Los registros de presencia y ausencia de las especies fueron usados para generar una matriz que se transformó en valores de similitud. Posteriormente estos valores fueron empleados para generar un dendrograma que ilustra los patrones de similitud florística; para el agrupamiento se aplicó el método de enlace completo. La misma matriz de presencias-ausencias se utilizó también para priorizar, mediante un método heurístico de complementariedad (Villaseñor et al., 2003), los cuadros que contribuyen de manera más eficiente con una estrategia de conservación de la riqueza florística. 


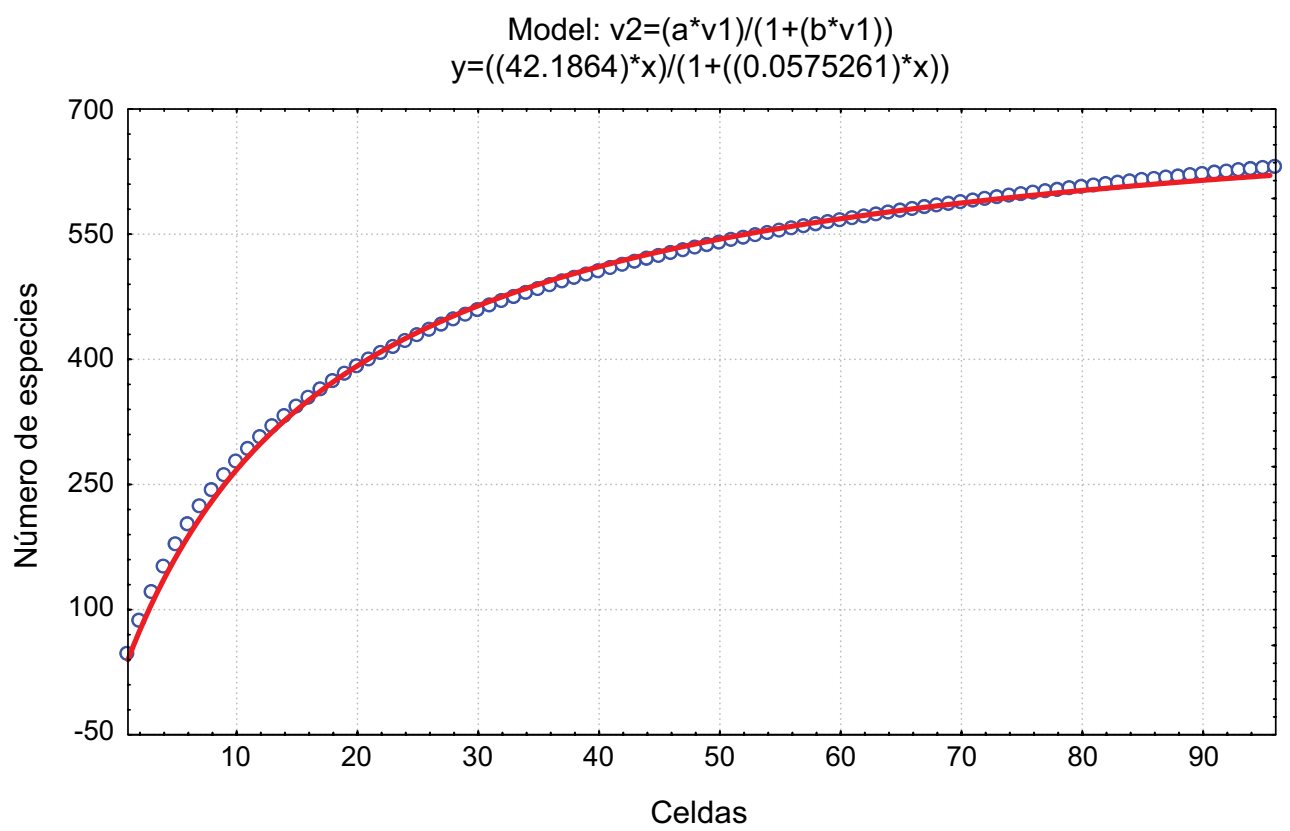

Fig. 3. Curva de acumulación de especies de Asteraceae en la región del Bajío. Los círculos representan las unidades de muestreo (cuadros de $0.25^{\circ} \times 0.25^{\circ}$ ). Los parámetros de la curva se indican en la ecuación de la parte superior de la figura. La asíntota se alcanza en las 773 especies, lo que indica un nivel de completitud de $88 \%$ en el conocimiento de las Asteraceae en la región.

\section{RESULTADOS}

Con base en material de herbario revisado, hasta ahora se ha documentado la presencia de 681 especies de Asteraceae (Apéndice), distribuidas en 182 géneros; de ellas 17 son especies no nativas, es decir, exóticas o introducidas. De la región de estudio, Querétaro es el estado que más especies registra (504), seguido por Michoacán (490) y Guanajuato (389).

El Cuadro 2 presenta un resumen de la distribución de los géneros y especies en las tribus en que actualmente se está dividiendo a las Asteraceae (Funk et al., 2009). En la región se encuentran 21 de las 24 tribus que contienen miembros de la familia en México; por otra parte, el número de géneros representa $50 \%$ de la riqueza genérica a nivel nacional y el de especies en la región de estudio constituye $23.1 \%$ de la cantidad global de especies. En promedio se encontró una proporción de 3.7 especies por género. 
Cuadro 2. Especies de Asteraceae registradas en la región del proyecto Flora del Bajío y de regiones adyacentes y su distribución por tribus.

\begin{tabular}{lcccc}
\hline Tribu & Géneros & Especies & $\begin{array}{c}\text { Especies endémicas de la } \\
\text { Región del Bají }\end{array}$ & $\begin{array}{c}\text { Especies } \\
\text { exóticas }\end{array}$ \\
\hline Anthemideae & 6 & 10 & & 6 \\
Astereae & 25 & 68 & 2 & \\
Bahieae & 5 & 8 & 1 & 2 \\
Cardueae & 4 & 20 & & 6 \\
Cichorieae & 8 & 14 & 4 & \\
Coreopsideae & 7 & 51 & & \\
Eupatorieae & 22 & 123 & & \\
Gnaphalieae & 3 & 19 & & \\
Gochnatieae & 1 & 2 & & \\
Helenieae & 1 & 3 & & \\
Heliantheae & 45 & 144 & & \\
Inuleae & 2 & 3 & & \\
Liabeae & 1 & 2 & & \\
Millerieae & 15 & 52 & & \\
Mutisieae & 2 & 6 & 3 & \\
Nassauvieae & 3 & 32 & & \\
Neurolaeneae & 2 & 3 & & \\
Perityleae & 3 & 4 & & \\
Senecioneae & 10 & 62 & & \\
Tageteae & 9 & 35 & & \\
Vernonieae & 8 & 20 & & \\
& 182 & 681 & & \\
\hline
\end{tabular}

Poco más de la mitad de las especies de Asteraceae registradas en la región son endémicas de México (55.9\%). De ellas, 29 solamente se conocen restringidas al territorio definido por el proyecto Flora del Bajío y de regiones adyacentes (endémicas locales). Para el estado de Guanajuato se conocen cuatro de estas endémicas locales, para Michoacán cinco, para Querétaro 15 y cinco se distribuyen en territorio tanto de Guanajuato como de Querétaro (Apéndice).

Es de esperar que en zonas como la aquí estudiada, con más de la mitad de la superficie original transformada, el número de especies sinantrópicas (asociadas al disturbio antropocéntrico) sea alto. En total se reconocen 358 como malezas, lo que 
representa $53.1 \%$ de la riqueza conocida. En el Cuadro 3 se indica la distribución de estas especies por cuadro; es evidente que mientras más superficie transformada contiene el cuadro, se registra un mayor número de malezas. Por ejemplo, el 14 tiene casi $75 \%$ de su territorio con vegetación natural transformada, tiene una diversidad registrada muy baja y la mayoría de las especies conocidas en la zona son malezas.

Esfuerzo de colecta

La evaluación del esfuerzo de colecta (Fig. 3) sugiere que en la región de estudio se deberían registrar 773 especies (valor de la asíntota de la curva de acumulación). En

Cuadro 3. Riqueza de Asteraceae en los 20 cuadros en que se dividió la región del proyecto Flora del Bajío y de regiones adyacentes.

\begin{tabular}{cccccc}
\hline Cuadro & $\begin{array}{c}\text { Superficie } \\
\left(\mathrm{km}^{2}\right)\end{array}$ & $\begin{array}{c}\text { Superficie con } \\
\text { vegetación } \\
\text { conservada (\%) }\end{array}$ & $\begin{array}{c}\text { Superficie sin } \\
\text { vegetación } \\
\text { aparente }(\%)\end{array}$ & Especies & Malezas (\%) \\
\hline 1 & 2179.0 & 52.6 & 47.4 & 23 & $20(87.0)$ \\
2 & 1481.9 & 49.2 & 50.8 & 44 & $36(81.8)$ \\
3 & 3110.6 & 61.4 & 38.6 & 141 & $99(70.2)$ \\
4 & 3742.5 & 41.6 & 58.4 & 96 & $73(76.0)$ \\
5 & 3901.2 & 75.4 & 24.6 & 157 & $104(66.2)$ \\
6 & 3081.1 & 87.2 & 12.8 & 225 & $139(61.8)$ \\
7 & 2271.3 & 84.7 & 15.3 & 175 & $116(66.7)$ \\
8 & 3154.6 & 23.6 & 76.4 & 45 & $36(80.0)$ \\
9 & 3137.1 & 29.1 & 70.9 & 78 & $61(78.2)$ \\
10 & 3136.6 & 31.8 & 68.2 & 121 & $99(81.8)$ \\
11 & 3138.1 & 44.2 & 55.8 & 176 & $121(68.7)$ \\
12 & 2879.5 & 66.3 & 33.7 & 200 & $124(62.0)$ \\
13 & 4054.7 & 27.5 & 72.5 & 85 & $71(83.5)$ \\
14 & 3163.0 & 25.2 & 74.8 & 53 & $49(92.4)$ \\
15 & 3163.9 & 24.0 & 76.0 & 131 & $107(81.7)$ \\
16 & 3675.2 & 21.1 & 78.9 & 198 & $147(74.2)$ \\
17 & 4596.2 & 45.8 & 54.2 & 262 & $171(65.3)$ \\
18 & 3212.3 & 37.6 & 62.4 & 261 & $177(68.3)$ \\
19 & 1604.4 & 41.0 & 59.0 & 257 & $161(62.6)$ \\
20 & 1467.4 & 29.1 & 70.9 & 231 & $150(64.6)$ \\
\hline & & & & &
\end{tabular}


consecuencia, si hasta la fecha se ha documentado la existencia de 681, el nivel de completitud de la flora bajo estudio es de $88 \%$. La revisión de la literatura indica la posible existencia de otras 66 en la región para las cuales aún no se ha verificado su presencia con ejemplares de herbario revisados; seguramente varias de ellas formarán parte del conjunto de casi 100 especies que de acuerdo con el modelo faltan por adicionar.

\section{Similitudes florísticas}

El análisis de agrupamiento permite identificar tres grupos principales (Fig. 4), uno de ellos concentra los cuadros ubicados en la porción occidental de la región de estudio (cuadros 2, 8, 13 y 14), caracterizados por una relativa pobreza de especies y un alto porcentaje de su superficie transformada por las actividades humanas. Aunque no se liga directamente a este grupo, el cuadro 1 seguramente también pertenece a esta unidad fitogeográfica. Otro conjunto comprende principalmente los ubicados en la porción central (cuadros $3,4,5,9,10,11,12$ y 15) y el otro asocia los localizados en los extremos este y sur de la zona de estudio (cuadros 6, 7, 16, 17,

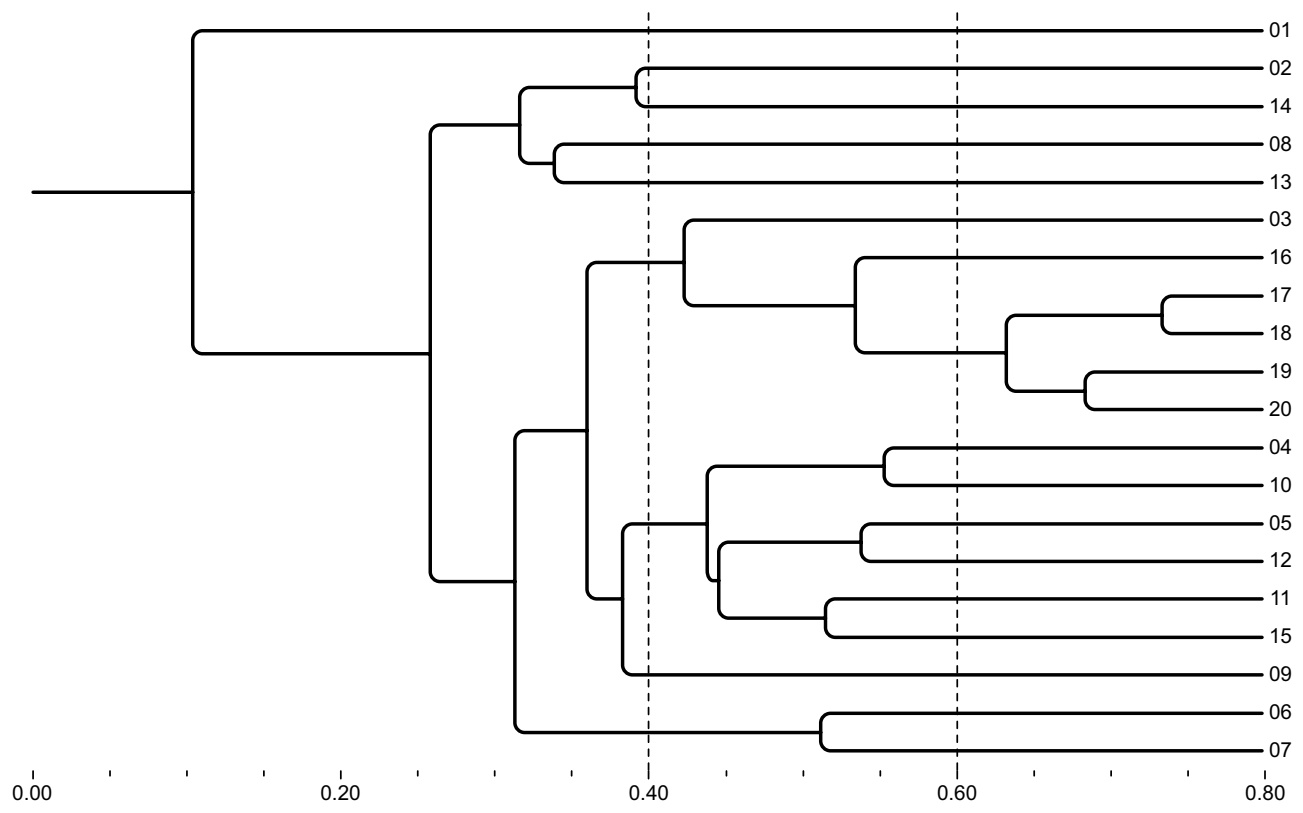

Fig. 4. Similitud florística entre los 20 cuadros en que se dividió la región que comprende el proyecto Flora del Bajío y de regiones adyacentes. Coeficiente de similitud utilizado: Sorensen-Dice; método de agrupamiento: enlace completo. 
18,19 y 20). Los agrupamientos señalan una tendencia a incrementar la riqueza de especies de oeste a este y de norte a sur, siguiendo un marcado contraste de heterogeneidad topográfica, de zonas más bien planas (primer grupo) a otra con accidentes geográficos aislados (por ejemplo la Sierra de Santa Rosa, ubicada principalmente en el cuadro 3) a zonas eminentemente accidentadas, como son las partes de la Faja Volcánica Transmexicana o la Sierra Madre Oriental, que conforman principalmente el tercer grupo. Los patrones geográficos revelados por el análisis de agrupamiento muestran una fuerte correspondencia con los de distribución de riqueza (Fig. 5).

Sitios importantes para la conservación de la riqueza florística

La figura 5 pone de manifiesto que la distribución de la riqueza de Asteraceae no es homogénea en la región de estudio; existen zonas con una relativa pobreza de especies y en otras se observa una considerable diversidad. La preocupación de

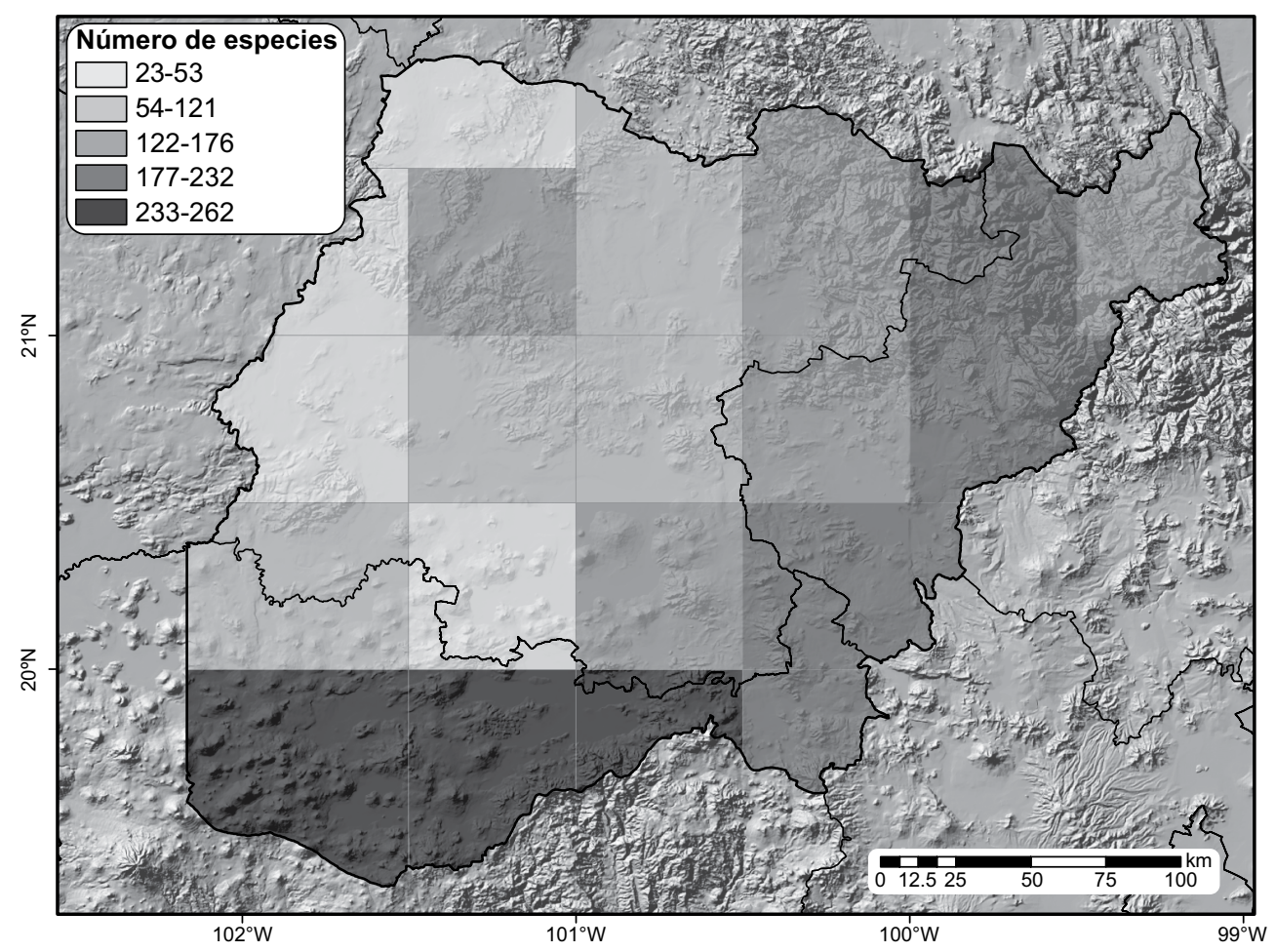

Fig. 5. Distribución de la riqueza de especies de Asteraceae en la región del Bajío. 
los biólogos de la conservación se centra principalmente en aquellas especies más proclives a la extinción, como son las raras o endémicas de una particular comarca. En la región de estudio más de la mitad (55.9\%) de sus especies son endémicas de México, lo que las hace candidatas a ser consideradas en las estrategias de conservación; sin embargo, un componente más importante en esta zona tan afectada por las actividades humanas lo constituyen sin lugar a dudas sus elementos endémicos locales, es decir, restringidos al territorio que abarca la zona del proyecto florístico. La distribución de las 29 especies endémicas locales (Cuadro 2, Apéndice) se encuentra registrada en 10 de los 20 cuadros en que se dividió la zona de estudio $(4,5$, $6,7,10,11,12,18,19$ y 20).

Un análisis de complementariedad, llevando a cabo un procedimiento heurístico iterativo (Villaseñor et al., 2003) y considerando solamente los 10 cuadros donde se ha registrado la presencia de las especies endémicas de la región de estudio, señala la siguiente prioridad en una jerarquía de selección de áreas si se pretendiera planificar una estrategia de conservación de este capital natural: cuadros 18, 6, 19, 7, $12,20,11,5,10$ y 4 . Con una estrategia de conservación en tales cuadros se podría proteger $94.1 \%$ de la riqueza de Asteraceae registrada en la región.

\section{DISCUSIÓN}

Las comunidades vegetales participan de manera importante en la conservación y manejo sustentable del ambiente en que vivimos. Por lo tanto, son uno de los dones más preciados que la naturaleza nos ha proporcionado, pues de allí obtenemos la gran parte de los bienes y servicios de los que depende nuestra subsistencia. Las especies vegetales, que en conjunto constituyen estas comunidades, representan un nivel de integración del conocimiento sobre la diversidad biológica que es básico para entender la estructura y funcionamiento de los ecosistemas. A partir de esta información se generan las estrategias de conservación de la biodiversidad y los planes para su manejo y aprovechamiento sustentable. El registro hasta ahora de 681 especies de Asteraceae en la región del Bajío y sus áreas adyacentes subraya la gran riqueza que la región alberga; por otra parte, su distribución geográfica permite identificar zonas particularmente ricas que servirán sin lugar a dudas para su evaluación como áreas de ayuda potencial en la conservación de la biodiversidad regional.

Existe una importante y significativa correlación entre la cantidad de especies de Asteraceae y la global de plantas vasculares que constituyen la flora de la región de estudio. Las estimaciones sugieren que por cada especie de Asteraceae existen al 
menos unas seis de otros grupos de plantas vasculares, entonces la riqueza florística total en la región consiste en más de 4000 especies. Además, para cada cuadro se estima una riqueza florística total en promedio de alrededor de más de 800 especies.

Los resultados de este análisis brindan una idea del camino a tomar para enfocar los futuros estudios dirigidos a proponer y reforzar las estrategias de conservación de esta flora regional. Aunque la mayoría de los cuadros tienen más de la mitad de su territorio con la vegetación autóctona perdida (Cuadro 2), todavía hay algunos que podrían servir como reservorios de la flora y vegetación original de la región. Tal es por ejemplo el caso del cuadro 3 en la parte noroeste de la zona de estudio, que al parecer todavía conserva más de $60 \%$ de su vegetación original y registra $18.9 \%$ de la riqueza de Asteraceae. Sin embargo, al parecer las áreas más ricas en especies y con vegetación conservada se restringen a los extremos meridional y oriental de la región, donde las partes montañosas están mejor representadas. La heterogeneidad ambiental allí encontrada seguramente limita un poco la extensión agrícola, urbana o industrial, que es más manifiesta en las planicies de la región central de la zona de estudio.

Los patrones de riqueza mostrados en la Figura 5 ayudan a generar ideas o a plantear hipótesis a evaluar en futuros estudios que permitan formular estrategias para la conservación de la diversidad florística de la región. Por ejemplo, es evidente que no es posible proponer una gran reserva allí por lo fragmentado de los parches de vegetación con poca perturbación antrópica; entonces, definir los sitios para establecer pequeñas reservas y corredores que conecten los fragmentos con mayor riqueza sería una estrategia factible. Los 10 cuadros donde se registran especies endémicas restringidas a la región de estudio (endémicas locales) y una alta riqueza de especies asociada a ellas (94.1\%) podrían contener un sistema regional de áreas de protección conectados que permitan el libre moviento de los organismos entre ellas. La adición de fragmentos en otros cuadros ayudaría a conservar prácticamente $100 \%$ de toda la riqueza conocida (por ejemplo en los cuadros 3 o 17). En este caso, la experiencia con mamíferos en México bien podría ser un paradigma a seguir para aplicarlo a las plantas de la región (Fuller et al., 2006).

En la región del Bajío se conjugan las principales amenazas a la biodiversidad. La zona se encuentra entre las más dinámicas en su crecimiento industrial, lo que provoca a se vez un incremento en la expansión humana. Por otra parte, la deforestación y el cambio de uso del suelo, especialmente para las actividades agrícolas también ha contribuido a la reducción de la cubierta vegetal original, llegando hasta nuestros días a un nivel de $56.4 \%$ de pérdida de la vegetación original. La evidencia científica y el sentido común nos llevan a pensar que de seguir esta tendencia sin 
minimizar su impacto negativo, la degradación continua del hábitat seguirá provocando un empobrecimiento de su biodiversidad y tal vez ya nunca lleguemos a saber cuál era su magnitud original.

Actualmente la sociedad está más preocupada por obtener del suelo el máximo beneficio posible, ya sea con agricultura extensiva, principalmente monocultivos o estableciendo industrias de transformación y la región del Bajío en México ilustra muy bien este fenómeno. Pero como el eminente ecólogo E. Odum (1969) decía, el hombre no sólo vive de comida y fibras, en el suelo además vivimos y convivimos con otros seres vivos, plantas y animales. ¿Hasta dónde podemos llevar, por ejemplo, la pérdida de la cobertura vegetal en la región del Bajío, antes de que los servicios ambientales que brinda la biodiversidad (recarga de acuíferos, reducción de la contaminación ambiental, evitar la erosión del suelo, por mencionar algunos) se pierdan totalmente, especialmente por nuestra ignorancia de que es la naturaleza quien nos brinda estos servicios? Las estimaciones de riqueza por unidad de área en la región sugieren que la zona es tan rica en diversidad vegetal como cualquier otra parte de México, en algunos sitios (sur y oriente por ejemplo) bastante por arriba de la media nacional. Solamente de nosotros depende mantener este capital natural para generaciones futuras, o soportar y arrepentirnos en unos pocos años de las catástrofes que el deterioro ambiental ocasione sobre nuestras actividades cotidianas, debido a la pérdida de estos sutiles pero imprescindibles servicios ambientales que la diversidad biológica nos brinda.

\section{AGRADECIMIENTOS}

Parte de los resultados aquí plasmados se obtuvieron con apoyo de la Comisión Nacional para el Conocimiento y Uso de la Biodiversidad (Conabio) y del Instituto de Biología de la Universidad Nacional Autónoma de México (proyecto SIBA). La Bióloga Guadalupe Segura ha colaborado ampliamente en la incorporación y manejo de la base de datos de donde se obtuvo la información para los análisis aquí presentados. El Doctor Gustavo Cruz revisó una versión preliminar y aportó importantes comentarios que enriquecieron el trabajo.

\section{LITERATURA CITADA}

Anónimo. 2005. Carta de uso del suelo y vegetación. Serie III. Escala 1:250,000. Instituto Nacional de Estadística y Geografía e Informática. Aguascalientes, México. 
Arita, H., F. Figueroa, A. Frisch, P. Rodríguez y K. Santos del Prado. 1997. Geographical range size and the conservation of Mexican mammals. Conserv. Biol. 11: 92-100.

Arreguín-Sánchez, M. de la L. y R. Fernández N. 2004. Flora de la Sierra Gorda, Querétaro. In: Luna, I., J. J. Morrone y D. Espinosa (eds.). Biodiversidad de la Sierra Madre Oriental. Las Prensas de Ciencias, Facultad de Ciencias, Universidad Nacional Autónoma de México y Comisión Nacional para el Conocimiento y Uso de la Biodiversidad. México, D.F., México. pp. 193-214.

Balleza, J. J., J. L. Villaseñor y G. Ibarra-Manríquez. 2005. Regionalización biogeográfica de Zacatecas, México, con base en los patrones de distribución de la familia Asteraceae. Rev. Mex. Biodiv. 76: 71-78.

Baltazar R., J. O., M. Martínez y Díaz y L. Hernández S. 2004. Guía de plantas comunes del Parque Nacional El Cimatario y sus alrededores. Tesis de licenciatura en Biología. Universidad Autónoma de Querétaro. Querétaro, México. 86 pp.

Cabrera L., J. A. y M. Gómez S. 2005. Análisis florístico de La Cañada, Querétaro, México. Bol. Soc. Bot. Méx. 77: 35-50.

Calderón de R., G. 1997. Familia Compositae, tribu Lactuceae. In: Rzedowski, J. y G. Calderón de Rzedowski (eds.). Flora del Bajío y de Regiones Adyacentes. Fascículo 54. Instituto de Ecología, A.C., Centro Regional del Bajío. Pátzcuaro, Michoacán, México. $55 \mathrm{pp}$.

Cartujano, S., S. Zamudio, O. Alcántara y I. Luna. 2002. El bosque mesófilo de montaña en el municipio de Landa de Matamoros, Querétaro, México. Bol. Soc. Bot. Méx. 70: 13-43.

Colwell, R. K. 2009. EstimateS, Version 8.2.0: statistical estimation of species richness and shared species from samples (Software and User's Guide). Freeware published at http://viceroy.eeb.uconn.edu/estimates.

Cornejo-Tenorio, G., A. Casas, B. Farfán, J. L. Villaseñor y G. Ibarra-Manríquez. 2003. Flora y vegetación de las zonas núcleo de la Reserva de la Biosfera Mariposa Monarca, México. Bol. Soc. Bot. Méx. 73: 43-62.

Espejo S., A., J. L. Brunhuber M., G. Segura W. y J. Ibarra C. 1992. La vegetación de la zona de hibernación de la Mariposa Monarca (Danaus plexippus L.) en la Sierra Chincua. In: Darwin, S. P. y A. L. Welden (eds.). Biogeography of Mesoamerica. Proceedings of a Symposium. Tulane Studies in Zoology and Botany. Supplementary Publication 1: 79-99.

Espinosa G., J. y L. S. Rodríguez-Jiménez. 1995. Listado florístico del estado de Michoacán. Sección II(Angiospermae: Compositae). In: Rzedowski, J. y G. Calderón de Rzedowski (eds.). Flora del Bajío y de Regiones Adyacentes. Fascículo complementario VII. Instituto de Ecología, A.C., Centro Regional del Bajío. Pátzcuaro, Michoacán, México. $242 \mathrm{pp}$.

Espinosa G., J y L. S. Rodríguez-Jiménez. 1996. Listado florístico del estado de Michoacán. Sección IV (Angiospermae: Fagaceae, Gramineae, Krameriaceae, Leguminosae). In: Rzedowski, J. y G. Calderón de Rzedowski (eds.). Flora del Bajío y de Regiones Adyacentes. Fascículo complementario XII. Instituto de Ecología, A.C., Centro Regional del Bajío. Pátzcuaro, Michoacán, México. 271 pp.

Fernández N., R. y A. Colmenero R. 1997. Notas sobre la vegetación y flora del municipio de San Joaquín, Querétaro, México. Polibotánica 4: 10-36. 
Fuller, T., M. Munguía, M. Mayfield, V. Sánchez-Cordero y S. Sarkar. 2006. Incorporating connectivity into conservation planning: a multi-criteria case study from central Mexico. Biol. Conserv. 133: 131-142.

Funk, V. A., A. Sussana, T. F. Stuessy y R. J. Bayer (eds.). 2009. Systematics, evolution, and biogeography of Compositae. International Association for Plant Taxonomy. Vienna, Austria. 965 pp.

García L., E. y S. D. Koch. 1995. Familia Compositae, tribu Cardueae. In: Rzedowski, J. y G. Calderón de Rzedowski (eds.). Flora del Bajío y de Regiones Adyacentes. Fascículo 32. Instituto de Ecología, A.C., Centro Regional del Bajío. Pátzcuaro, Michoacán, México. $51 \mathrm{pp}$.

Gómez S., M. y L. Sánchez C. 1999. Flora vascular del Cerro El Zamorano. Informe final. Sistema Nacional de Información sobre Biodiversidad de México-Comisión Nacional para el Conocimiento y Uso de la Biodiversidad proyecto L002. México, D.F., México. http://www.conabio.gob.mx/institucion/proyectos/resultados/InfL002. pdf.

Gotelli, N. J. y R. K. Colwell. 2001. Quantifying biodiversity: procedures and pitfalls in the measurement and comparison of species richness. Ecol. Lett. 4: 379-391.

Ibarra- Manríquez, G., J. L. Villaseñor, R. Durán y J. Meave. 2002. Biogeographical analysis of the tree flora of Yucatan Peninsula. J. Biogeogr. 29: 17-29.

Jiménez-Valverde, A. y J. Hortal. 2003. Las curvas de acumulación de especies y la necesidad de evaluar la calidad de los inventarios biológicos. Rev. Ibér. Arac. 8: 151-161.

Lot, A. y A. Novelo. 1988. Vegetación y flora acuática del Lago de Pátzcuaro, Michoacán, México. Southwest. Nat. 33: 167-175.

Martínez-Cruz, J. y O. Téllez-Valdés. 2004. Listado florístico de la Sierra de Santa Rosa, Guanajuato, México. Bol. Soc. Bot. Méx. 74: 31-49.

Martínez M., E., G. Ibarra C., A. Hernández V. y F. Lorea-Hernández. 1987. Contribución al conocimiento de la flora y la vegetación de la región de los Azufres, Michoacán. Trace 12: 22-37.

Meagher, W. L. 1994. Lista de la flora espontánea del Jardín Botánico "El Charco del Ingenio", San Miguel de Allende, Guanajuato (México). In: Rzedowski, J. y G. Calderón de Rzedowski (eds.). Flora del Bajío y de Regiones Adyacentes. Fascículo complementario V. Instituto de Ecología, A.C., Centro Regional del Bajío. Pátzcuaro, Michoacán, México. $36 \mathrm{pp}$.

Medina, C. y S. Rodríguez. 1993. Estudio florístico de la cuenca del Río Chiquito de Morelia, Michoacán, México. In: Rzedowski, J. y G. Calderón de Rzedowski (eds.). Flora del Bajío y de Regiones Adyacentes. Fascículo complementario IV. Instituto de Ecología, A.C., Centro Regional del Bajío. Pátzcuaro, Michoacán, México. $71 \mathrm{pp}$.

Mittermeier, R. A. 1988. Primate diversity and the tropical forest. In: Wilson, E. O. y F. M. Peter (eds.). Biodiversity. National Academic Press. Washington, D.C., USA. pp. 145-154.

Myers, N., R. A. Mittermeier, C. G. Mittermeier, G. A. B. da Fonseca y J. Kent. 2000. Biodiversity hotspots for conservation priorities. Nature 403: 853-858.

Odum, E. P. 1969. The strategy of ecosystem development. Science 164: 262-270. 
Pérez-Calix, E. 1996. Flora y vegetación de la cuenca del Lago de Zirahuén, Michoacán, México. In: Rzedowski, J. y G. Calderón de Rzedowski (eds.). Flora del Bajío y de Regiones Adyacentes. Fascículo complementario XIII. Instituto de Ecología, A.C., Centro Regional del Bajío. Pátzcuaro, Michoacán, México. 73 pp.

Quero, H. J. 1984. La vegetación de las serranías de la cuenca alta del Río de la Laja, Guanajuato. Anales Inst. Biol. Univ. Nac. México, Ser. Bot. 47-53: 73-99.

Rodríguez-Jiménez, L. S. y J. Espinosa G.1995. Listado florístico del estado de Michoacán. Sección I (Gymnospermae; Angiospermae: Acanthaceae-Commelinaceae). In: Rzedowski, J. y G. Calderón de Rzedowski (eds.). Flora del Bajío y de Regiones Adyacentes. Fascículo complementario VI. Instituto de Ecología, A.C., Centro Regional del Bajío. Pátzcuaro, Michoacán, México. 208 pp.

Rodríguez-Jiménez, L. S. y J. Espinosa G.1996a. Listado florístico del estado de Michoacán. Sección III (Angiospermae: Connaraceae-Myrtaceae, excepto Fagaceae, Gramineae, Krameriaceae y Leguminosae). In: Rzedowski, J. y G. Calderón de Rzedowski (eds.). Flora del Bajío y de Regiones Adyacentes. Fascículo complementario X. Instituto de Ecología, A.C., Centro Regional del Bajío. Pátzcuaro, Michoacán, México. 296 pp.

Rodríguez-Jiménez, L. S. y J. Espinosa G.1996b. Listado florístico del estado de Michoacán. Sección V (Angiospermae: Najadaceae-Zygophyllaceae). In: Rzedowski, J. y G. Calderón de Rzedowski (eds.). Flora del Bajío y de Regiones Adyacentes. Fascículo complementario XV. Instituto de Ecología, A.C., Centro Regional del Bajío. Pátzcuaro, Michoacán, México. 344 pp.

Rzedowski, J. 1991. Diversidad y orígenes de la flora fanerogámica de México. Acta Bot. Mex. 14: 3-21.

Rzedowski, J. y G. Calderón de R. 1997. Familia Compositae, tribu Anthemideae. In: Rzedowski, J. y G. Calderón de Rzedowski (eds.). Flora del Bajío y de Regiones Adyacentes. Fascículo 60. Instituto de Ecología, A.C., Centro Regional del Bajío. Pátzcuaro, Michoacán, México. 29 pp.

Rzedowski, J. y G. Calderón de R. 2008. Familia Compositae, tribu Heliantheae I. In: Rzedowski, J. y G. Calderón de Rzedowski (eds.). Flora del Bajío y de Regiones Adyacentes. Fascículo 157. Instituto de Ecología, A.C., Centro Regional del Bajío. Pátzcuaro, Michoacán, México. 344 pp.

Rzedowski, J., G. Calderón de R. y P. Carrillo-Reyes. 2011. Familia Compositae, tribu Heliantheae II. In: Rzedowski, J. y G. Calderón de Rzedowski (eds.). Flora del Bajío y de Regiones Adyacentes. Fascículo 172. Instituto de Ecología, A.C., Centro Regional del Bajío. Pátzcuaro, Michoacán, México. 409 pp.

Villarreal Q., J. A., J. L. Villaseñor y R. Medina L. 2006. Familia Compositae, tribu Helenieae. In: Rzedowski, J. y G. Calderón de Rzedowski (eds.). Flora del Bajío y de Regiones Adyacentes. Fascículo 140. Instituto de Ecología, A.C., Centro Regional del Bajío. Pátzcuaro, Michoacán, México. 54 pp.

Villaseñor, J. L. 2003. Diversidad y distribución de las Magnoliophyta de México. Interciencia 28: 160-167.

Villaseñor, J. L., J. A. Meave, E. Ortíz y G. Ibarra-Manríquez. 2003. Biogeografía y conservación de los bosques tropicales húmedos de México. In: Morrone, J. J. y J. Llorente B. (eds.). Una perspectiva Latinoamericana de la Biogeografía. Comisión Nacional para el Conocimiento y Uso de la Biodiversidad y Universidad Nacional Autónoma de México. México, D.F., México. pp. 209-216. 
Villaseñor, J. L., P. Maeda, J. A. Rosell y E. Ortiz. 2007. Plant families as predictors of plant biodiversity in Mexico. Divers. Distrib. 13: 871-876.

Zamudio, S. 1984. La vegetación de la cuenca del Río Estórax, en el estado de Querétaro y sus relaciones fitogeográficas. Tesis de licenciatura. Facultad de Ciencias, Universidad Nacional Autónoma de México. México, D.F., México. 275 pp.

Recibido en marzo de 2012.

Aceptado en junio de 2012. 


\section{APÉNDICE}

Lista de especies de Asteraceae registradas para la región que comprende el proyecto Flora del Bajío y de regiones adyacentes (Fig. 1). Con un asterisco (*) se indican las introducidas a la flora de México. Los estados se indican con abreviaciones y los números hacen referencia a la posición indicada en la Figura 1.

Tribu Anthemideae

Achillea millefolium L. GTO, MICH, QRO; 6, 11, $13,19,20$

*Artemisia absinthium L. GTO, MICH, QRO; 3, 16,18

Artemisia klotzschiana Besser GTO, QRO; 4 Artemisia ludoviciana Nutt. GTO, MICH, QRO; $1,3,4,5,9,11,12,15,16,18,19,20$

*Cotula australis (Spreng.) Hook. f. MICH, QRO; 7, 17

Cotula mexicana (DC.) Cabrera MICH, QRO; 19,20

*Leucanthemum lacustre (Brot.) Samp. MICH; 19

*Soliva anthemifolia (Juss.) Sweet GTO, MICH; 1,18

*Soliva sessilis Ruiz \& Pav. MICH; 19, 20

*Tanacetum parthenium (L.) Sch. Bip. GTO, MICH, QRO; 11, 16, 17, 18, 20

\section{Tribu Astereae}

Almutaster pauciflorus (Nutt.) A. Löve \& D. Löve GTO, MICH, QRO; 11, 15, 16, 18

Aphanostephus ramosissimus DC. GTO, MICH, QRO; 3, 4, 5, 10, 12, 15, 16, 17, 19

Archibaccharis asperifolia (Benth.) S.F. Blake MICH; 17, 18, 19, 20

Archibaccharis auriculata (Hemsl.) G.L. Nesom GTO, MICH, QRO; 3, 6, 16

Archibaccharis hieracioides (S.F. Blake) S.F. Blake GTO, MICH; 18, 19, 20

Archibaccharis hirtella (DC.) Heering MICH, QRO; 17, 18, 19, 20

Archibaccharis schiedeana (Benth.) J.D. Jacks. $\mathrm{MICH}, \mathrm{QRO} ; 6,7,17,20$

Archibaccharis serratifolia (Kunth) S.F. Blake GTO, MICH, QRO; 3, 5, 6, 7, 12, 13, 16, 17, 18, 19, 20

Arida riparia (Kunth) D.R. Morgan \& R.L.

Hartm. GTO, MICH; 14, 18, 20
Astranthium laetificum De Jong MICH; 19 (endémica de la región)

Astranthium orthopodum (B.L. Rob. \& Fernald) Larsen GTO, MICH; 17, 18, 19

Astranthium purpurascens (B.L. Rob.) Larsen GTO, QRO; 4, 6, 7, 11, 12

Aztecaster matudae (Rzed.) G.L. Nesom QRO; 12

Baccharis brevipappa (McVaugh) G.L. Nesom $\mathrm{MICH} ; 17$

Baccharis conferta Kunth GTO, MICH, QRO; 5, 6, 7, 12, 16, 19, 20

Baccharis heterophylla Kunth GTO, MICH, QRO; 2, 3, 6, 16, 17, 18, 19, 20

Baccharis lancifolia Schltdl. QRO; 6,7

Baccharis multiflora Kunth GTO, MICH, QRO; $3,6,7,13,16,17,18,19,20$

Baccharis pteronioides DC. GTO, MICH, QRO; 1, 3, 5, 6, 10, 11, 12, 16, 17, 18, 19, 20

Baccharis ramiflora A. Gray GTO, QRO; 5, 11, 12

Baccharis salicifolia (Ruiz \& Pav.) Pers. GTO, MICH, QRO; 3, 4, 5, 6, 9, 10, 11, 13, 16, 17, 18, 19,20

Baccharis sordescens DC. GTO, MICH, QRO; 5, 11, 12, 14, 15, 18

Baccharis sulcata DC. GTO; 3

Baccharis thesioides Kunth GTO, MICH, QRO; 2, 3, 9, 16, 17, 18, 19

Baccharis trinervis (Lam.) Pers. MICH, QRO; 7

Baccharis zamoranensis Rzed. GTO, QRO; 11 (endémica de la región)

Conyza bonariensis (L.) Cronquist GTO, MICH, QRO; $12,15,18$

Conyza canadensis (L.) Cronquist GTO, MICH, QRO; 3, 11, 12, 17, 18, 19, 20

Conyza coronopifolia Kunth GTO, MICH, QRO; $13,16,17,18,19,20$

Conyza laevigata (Rich.) Pruski MICH, QRO; 6,7 
Apéndice. Continuación.

Conyza microcephala Hemsl. GTO, MICH, QRO; $10,12,15,16,17,18,20$

Chaetopappa ericoides (Torr.) G.L. Nesom GTO, QRO; 3, 4, 5, 6, 11, 12

Chloracantha spinosa (Benth.) G.L. Nesom GTO, MICH, QRO; 10, 11, 12, 15, 19

Erigeron delphinifolius Willd. GTO, MICH, QRO; 2, 3, 5, 6, 11, 13, 14, 15, 16, 17, 18, 19, 20

Erigeron galeottii (A. Gray ex Hemsl.) Greene GTO, MICH, QRO; 11, 20

Erigeron janivultus G.L. Nesom GTO, MICH, QRO; 1, 2, 16

Erigeron karvinskianus DC. GTO, MICH, QRO; $6,7,18,19,20$

Erigeron longipes DC. GTO, MICH, QRO; 3, 6, $7,10,11,12,13,15,16,17,18,19,20$

Erigeron polycephalus (Larsen) G.L. Nesom $\mathrm{MICH} ; 19$

Erigeron pubescens Kunth GTO, MICH, QRO; $3,6,12$

Erigeron velutipes Hook. \& Arn. GTO, MICH, QRO; 16, 17, 18, 19

Grindelia inuloides Willd. GTO, MICH, QRO; 6, 18

Grindelia nelsonii Steyerm. MICH, 17

Grindelia oxylepis Greene GTO, QRO; 16

Grindelia subdecurrens DC. GTO, MICH, QRO; $3,6,10$

Gutierrezia sericocarpa (A. Gray) M.A. Lane GTO, QRO; 3, 5, 16

Gutierrezia texana (DC.) Torr. \& A. Gray GTO, QRO; 4, 5, 6, 10

Gymnosperma glutinosum (Spreng.) Less. GTO, QRO; 1, 4, 5, 10, 12, 15

Heterotheca inuloides Cass. GTO, MICH, QRO; $16,17,19$

Heterotheca leptoglossa DC. GTO, QRO; 2, 3, 4, $5,9,10,11,16,19$

Isocoma hartwegii (A. Gray) Greene GTO; 1, 3, 4

Laennecia confusa (Cronquist) G.L. Nesom GTO, MICH, QRO; 5, 9, 18

Laennecia filaginoides DC. GTO, MICH, QRO; $3,5,9,16,17,18,19,20$

Laennecia schiedeana (Less.) G.L. Nesom GTO, MICH, QRO; 11, 13, 15, 16, 17, 18, 20

Laennecia sophiifolia (Kunth) G.L. Nesom GTO, MICH, QRO; 2, 5, 13, 16, 17, 18, 19, 20
Olivaea tricuspis Sch. Bip. ex Benth. GTO, $\mathrm{MICH} ; 17$

Osbertia stolonifera (DC.) Greene MICH; 20

Psilactis asteroides A. Gray GTO, MICH, QRO; $1,5,11,14,15,16,17,18,19$

Psilactis brevilingulata Sch. Bip. ex Hemsl. GTO, MICH, QRO; 5, 10, 11, 13, 17, 18, 19, 20

Psilactis gentryi (Standl.) D.R. Morgan QRO; 11, 16

Solidago simplex Kunth GTO, QRO; 5

Symphyotrichum expansum (Poepp. ex Spreng.) G.L. Nesom GTO, MICH, QRO; 3, 5, 7, 10, 11, $12,13,15,16,17,18,19,20$

Symphyotrichum moranense (Kunth) G.L. Nesom GTO, MICH, QRO; 3, 6, 16, 17, 18, 19, 20

Symphyotrichum trilineatum (Sch. Bip. ex Klatt) G.L. Nesom GTO; 4

Townsendia mexicana A. Gray MICH, QRO; 12 Xanthisma gymnocephalum (DC.) D.R. Morgan \& R.L. Hartm. GTO, QRO; 10, 16

Xanthisma spinulosum (Pursh) D.R. Morgan \& R.L. Hartm. GTO, QRO; 3, 4, 5, 10, 12

Xanthocephalum benthamianum Hemsl. GTO, $\mathrm{MICH} ; 17$

\section{Tribu Bahieae}

Achyropappus anthemoides Kunth GTO, QRO; $5,11,12$

Bahia absinthifolia Benth. GTO, QRO; 4, 5, 10, 12

Bahia pringlei Greenm. GTO, QRO; 4, 12

Bahia schaffneri $\mathrm{S}$. Watson GTO; 1, 3, 4, 5

Florestina pedata (Cav.) Cass. GTO, MICH, QRO; 3, 4, 5, 6, 8, 9, 10, 11, 12, 13, 14, 15, 16, $17,18,19$

Loxothysanus pedunculatus Rydb. QRO; 7

Schkuhria pinnata (Lam.) Kuntze ex Thell. GTO, MICH, QRO; 2, 3, 4, 5, 8, 10, 11, 12, 13, 14, 15, $16,17,18,19,20$

Schkuhria schkuhrioides (Link. \& Otto) Thell. GTO, MICH; 4, 9, 10, 13, 15, 18, 19

\section{Tribu Cardueae}

*Carthamus tinctorius L. GTO, MICH; 11, 18

Centaurea americana Nutt. QRO; 7, 16

Centaurea rothrockii Greenm. MICH; 17 
Apéndice. Continuación.

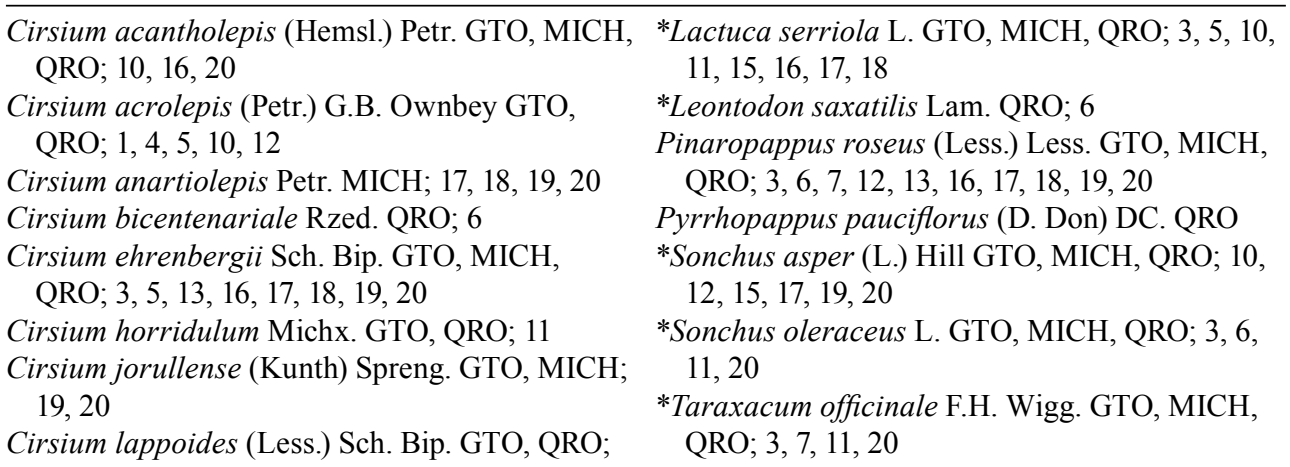

$$
5,6,7,16
$$

Cirsium mexicanum DC. GTO, QRO; 7, 11

Cirsium pascuarense (Kunth) Spreng. $\mathrm{MICH} ; 17$, 18

Cirsium pinetorum Greenm. GTO, MICH, QRO; 7, 11,12

Cirsium rhaphilepis (Hemsl.) Petr. GTO, MICH, QRO; 9, 10, 11, 12, 13, 15, 16, 17, 18

Cirsium subcoriaceum (Less.) Sch. Bip. GTO, MICH, QRO; 7, 17, 18, 19, 20

Cirsium tolucanum (B.L. Rob. \& Seaton) Petr. $\mathrm{MICH} ; 13,17$

Cirsium velatum (S. Watson) Petr. GTO, $\mathrm{MICH}$; 17, 18, 19, 20

Cirsium zamoranense Rzed. GTO, QRO; 11 (endémica de la región)

*Cynara cardunculus L. GTO, MICH, QRO; 6, $14,15,17$

\section{Tribu Cichorieae}

*Helminthotheca echioides (L.) Holub GTO, MICH, QRO; 10, 11, 18, 19

Hieracium abscissum Less. GTO, MICH, QRO; $3,5,6,7,11,13,16,17,18,19,20$

Hieracium crepidispermum Fries GTO, MICH, QRO; 11, 15, 17, 18, 19, 20

Hieracium dysonymum S.F. Blake GTO, MICH, QRO; 6, 18, 19, 20

Hieracium fendleri Sch. Bip. GTO, MICH, QRO; $11,18,19$

Hieracium schultzii Fr. GTO, MICH, QRO; 6, 11, 16

Lactuca graminifolia Michx. GTO, MICH, QRO; 7,12

\section{Tribu Coreopsideae}

Bidens aequisquama (Fernald) Sherff GTO, MICH, QRO; 3, 6, 17, 18, 20

Bidens alba (L.) DC. MICH, QRO; 6, 7

Bidens angustissima Kunth GTO, MICH, QRO; $3,9,11,12$

Bidens anthemoides (DC.) Sherff MICH; 19, 20

Bidens aurea (Aiton) Sherff GTO, MICH, QRO; $3,9,10,11,15,16,17,18,19,20$

Bidens bigelovii A. Gray GTO, MICH, QRO; 11, $15,17,18,19,20$

Bidens clavata R.E. Ballard QRO; 6, 7

Bidens ferulifolia (Jacq.) DC. GTO, MICH, QRO; 3, 4, 5, 10, 12, 15

Bidens laevis (L.) Britton, Sterns \& Pogg. GTO, MICH; 14, 17, 18, 19, 20

Bidens lemmonii A. Gray GTO, MICH; 2, 3, 19, 20

Bidens leptocephala Sherff QRO; 12

Bidens mollifolia Sherff MICH; 19

Bidens odorata Cav. GTO, MICH, QRO; 2, 3, 4, $5,6,10,11,12,14,15,16,17,18,19,20$

Bidens ostruthioides (DC.) Sch. Bip. GTO, MICH, QRO; 16, 17, 18, 19, 20

Bidens pilosa L. GTO, MICH, QRO; 4, 6, 7, 11, $12,14,15,16,17,18,19$

Bidens schaffneri (A. Gray) Sherff GTO, QRO; 10,12

Bidens serrulata (Poir.) Desf. GTO, MICH, QRO; $15,16,17,19$

Bidens squarrosa Kunth MICH, QRO; 7

Bidens triplinervia Kunth GTO, MICH, QRO; 5 , $6,7,12,19,20$ 
Apéndice. Continuación.

Chrysanthellum indicum DC. MICH, QRO; 16, 17,18

Chrysanthellum keilii B.L. Turner MICH; 17

Coreopsis crawfordii Mesfin QRO; 11 (endémica de la región)

Coreopsis guanajuatensis B.L. Turner GTO, QRO; 5, 10, 11 (endémica de la región)

Coreopsis mutica DC. GTO, MICH, QRO; 3, 7, 11,12

Coreopsis paludosa M.E. Jones GTO; 5

Coreopsis petrophiloides B.L. Rob. \& Greenm. GTO, MICH; 17, 18, 19, 20

Coreopsis queretarensis B.L. Turner QRO; 6 (endémica de la región)

Coreopsis rhyacophila Greenm. GTO, MICH, QRO; 11, 12, 16

Cosmos atrosanguineus (Ortgies) Hemsl. GTO, QRO; 5, 6, 7

Cosmos bipinnatus Cav. GTO, MICH, QRO; 3, 4, $5,6,8,9,10,11,12,14,15,16,17,18,19,20$

Cosmos carvifolius Benth. MICH; 17

Cosmos crithmifolius Kunth GTO, MICH, QRO; $3,5,8,9,12,17,18,19$

Cosmos diversifolius Otto GTO, MICH, QRO; 5, $6,7,11,12,19,20$

Cosmos palmeri B.L. Rob. GTO; 2, 3

Cosmos parviflorus (Jacq.) Pers. GTO, MICH, QRO; 3, 5, 6, 8, 9, 11, 12, 15, 16, 17, 18, 19, 20

Cosmos scabiosoides Kunth GTO, MICH; 17, 18, 19,20

Cosmos sulphureus Cav. GTO, MICH, QRO; 6, $7,8,17$

Dahlia coccinea Cav. GTO, MICH, QRO; 3, 4, 5 , $6,7,9,10,11,12,13,16,17,18,19,20$

Dahlia cuspidata Saar, P.D. Sorensen \& Hjert. GTO, QRO; 4, 6, 10, 12

Dahlia dissecta S. Watson QRO; 6, 12

Dahlia linearis Sherff GTO, QRO; 4, 10, 11 (endémica de la región)

Dahlia merckii Lehm. GTO, MICH, QRO; 6, 7, 9, $11,16,20$

Dahlia mollis P.D. Sorensen GTO, QRO; 6

Dahlia moorei Sherff QRO; 6, 11, 12

Dahlia neglecta Saar GTO, MICH, QRO; 11, 17, 18

Dahlia rudis P.D. Sorensen MICH; 20
Dahlia scapigera (A. Dietr.) Knowles \& Westc. GTO, MICH; 17, 18, 19, 20

Dahlia scapigeroides Sherff GTO, QRO; 6, 7, 11, 12

Dahlia sorensenii H.V. Hansen \& Hjert. GTO, MICH, QRO; 16, 17, 19, 20

Dicranocarpus parviflorus A. Gray QRO; 12 Heterosperma pinnatum Cav. GTO, MICH, QRO; $1,2,3,4,5,7,10,11,12,14,15,16,17,18,19,20$

\section{Tribu Eupatorieae}

Ageratina adenophora (Spreng.) R.M. King \& H. Rob. GTO, MICH, QRO; 6, 12, 15, 16, 18, 19,20

Ageratina areolaris (DC.) Gage GTO, MICH, QRO; 15, 16, 17, 18, 19, 20

Ageratina atrocordata (B.L. Rob.) R.M. King \&

H. Rob. GTO, MICH, QRO

Ageratina brandegeana (B.L. Rob.) R.M. King \&

H. Rob. GTO; 5

Ageratina brevipes (DC.) R.M. King \& H. Rob. GTO, MICH, QRO; 3, 4, 9, 10, 12, 13, 15, 16, $17,18,20$

Ageratina calaminthifolia (Kunth) R.M. King \& H. Rob. GTO, QRO; 3 Ageratina calophylla (Greene) R.M. King \& H.

Rob. GTO, QRO; 4, 5, 6, 10, 12, 16 Ageratina cardiophylla (B.L. Rob.) R.M. King \& H. Rob. GTO, MICH; 3

Ageratina chiapensis (B.L. Rob.) R.M. King \& H. Rob. MICH; 17

Ageratina choricephala (B.L. Rob.) R.M. King \& H. Rob. GTO, MICH, QRO; 16, 17

Ageratina conspicua (Kunth \& C.D. Bouché)

R.M. King \& H. Rob. GTO, MICH, QRO; 6, 7, $11,19,20$

Ageratina crassiramea (B.L. Rob.) R.M. King \& H. Rob. MICH; 19

Ageratina deltoidea (Jacq.) R.M. King \& H. Rob. GTO, MICH, QRO; 3, 13, 15, 16, 17, 20

Ageratina dolichobasis (McVaugh) R.M. King \& H. Rob. MICH; 19 Ageratina espinosarum (A. Gray) R.M. King \& H. Rob. GTO, QRO; 3, 5, 6, 10, 11, 12 Ageratina glabrata (Kunth) R.M. King \& H. Rob. GTO, MICH, QRO; 3, 4, 6, 11, 12, 16, 17, 18, 19,20 
Apéndice. Continuación.

Ageratina havanensis (Kunth) R.M. King \& H. Rob. GTO, QRO; 5, 6, 7, 16 Ageratina hidalgensis (B.L. Rob.) R.M. King \& H. Rob. QRO; 6, 11, 12

Ageratina isolepis (B.L. Rob.) R.M. King \& H. Rob. MICH, QRO; 6, 19, 20

Ageratina lasia (B.L. Rob.) R.M. King \& H. Rob. MICH; 18

Ageratina ligustrina (DC.) R.M. King \& H. Rob. GTO, MICH, QRO; 5, 7, 12

Ageratina mairetiana (DC.) R.M. King \& H. Rob. GTO, MICH, QRO; 3, 6, 17, 18, 19, 20

Ageratina malacolepis (B.L. Rob.) R.M. King \& H. Rob. MICH; 17

Ageratina muelleri (Sch. Bip. ex Klatt) R.M. King \& H. Rob. GTO, MICH; 5, 15, 16, 17, 18, 19 Ageratina oligocephala (DC.) R.M. King \& H. Rob. GTO, MICH, QRO; 7, 16, 17, 18, 19 Ageratina oreithales (Greenm.) B.L. Turner MICH, QRO; 6

Ageratina parayana (J. Espinosa) B.L. Turner $\mathrm{MICH} ; 19$

Ageratina pazcuarensis (Kunth) R.M. King \& H.

Rob. GTO, MICH, QRO; 5, 6, 11, 12, 15, 17, 19, 20 Ageratina petiolaris (Moc. \& Sessé ex DC.) R.M.

King \& H. Rob. GTO, MICH, QRO; 3, 5, 6, 7, 12, 16, 17, 18, 19, 20

Ageratina pichinchensis (Kunth) R.M. King \& H.

Rob. GTO, MICH, QRO; 6, 17, 19

Ageratina prunellifolia (Kunth) R.M. King \& H.

Rob. MICH, QRO; 11, 17

Ageratina queretaroana B.L. Turner QRO; 6

Ageratina ramireziorum (J. Espinosa) B.L. Turner MICH; 17, 19, 20

Ageratina rhomboidea (Kunth) R.M. King \& H.

Rob. GTO, MICH, QRO; 16, 17, 20

Ageratina rubricaulis (Kunth) R.M. King \& H.

Rob. GTO, MICH; 16, 17, 20

Ageratina saltillensis (B.L. Rob.) R.M. King \& H

Rob. QRO; 12

Ageratina scorodonioides (A. Gray) R.M. King \&

H. Rob. GTO, QRO; 4, 5, 10, 12

Ageratina viburnoides (DC.) R.M. King \& H.

Rob. QRO; 7

Ageratina wrightii (A. Gray) R.M. King \& H.

Rob. GTO, QRO
Ageratum corymbosum Zuccagni GTO, MICH, QRO; 3, 4, 9, 10, 11, 12, 15, 16, 17, 18, 20

Ageratum houstonianum Mill. QRO; 7

Brickellia diffusa (Vahl) A. Gray GTO, MICH, QRO; 6, 7

Brickellia eupatorioides (L.) Shinners GTO, MICH, QRO; 1, 4, 10, 15, 16, 18, 19

Brickellia glandulosa (La Llave) McVaugh GTO, MICH, QRO; 5, 6, 7, 17

Brickellia monocephala B.L. Rob. GTO, MICH; 10, 13, 17, 18

Brickellia nutanticeps S.F. Blake GTO, MICH, QRO; 6, 11, 16, 17, 19, 20

Brickellia oliganthes (Less.) A. Gray GTO, MICH, QRO; 3, 7, 18

Brickellia paniculata (Mill.) B.L. Rob. GTO, MICH, QRO; 5, 6, 19

Brickellia pavonii (A. Gray) B.L. Turner GTO, MICH, QRO; 7

Brickellia pedunculosa (DC.) Harc. \& Beaman GTO, MICH, QRO; 3, 5, 9, 16, 17, 18, 19, 20

Brickellia pendula (Schrad.) A. Gray GTO, MICH, QRO; 17, 18, 19, 20

Brickellia scoparia (DC.) A. Gray GTO, MICH, QRO; 5, 6, 12, 15

Brickellia secundiflora (Lag.) A. Gray GTO, MICH, QRO; 2, 3, 4, 5, 7, 8, 9, 10, 11, 13, 15, 16, 17, 18, 19, 20

Brickellia squarrosa B.L. Rob. \& Seaton MICH; 17, 18, 19

Brickellia subuligera (Schauer) B.L. Turner GTO, MICH, QRO; 6, 7, 11, 12, 15

Brickellia tomentella A. Gray GTO, QRO; 5, 8, 11

Brickellia veronicifolia (Kunth) A. Gray GTO, MICH, QRO; 5, 6, 7, 11, 12, 14, 16, 20

Carminatia alvarezii Rzed. \& Calderón MICH, QRO; 6, 7

Carminatia recondita $\mathrm{McVaugh} \mathrm{MICH}, \mathrm{QRO} ; 6$, $7,12,16,18,20$

Carminatia tenuiflora DC. GTO, MICH, QRO; $14,15,16,17,18,19,20$

Carphochaete grahamii A. Gray GTO, MICH, QRO; 3, 5, 9, 16, 17, 18, 19, 20

Chromolaena bigelovii (A. Gray) R.M. King \& H. Rob. QRO; 6 
Apéndice. Continuación.

Chromolaena collina (DC.) R.M. King \& H. Rob. Stevia clinopodioides Greenm. MICH; 20 GTO, MICH, QRO; 4, 5, 6, 7, 8, 11, 12, 15, 16, $17,18,19,20$

Chromolaena odorata (L.) R.M. King \& H. Rob. GTO, MICH, QRO; 5, 6, 7

Chromolaena pulchella (Kunth) R.M. King \& H. Rob. GTO, MICH, QRO; 10, 15, 16, 17, 18, 19,20

Conoclinium betonicifolium (Mill.) R.M. King \& H. Rob. GTO

Critonia daleoides DC. QRO; 7

Critonia lozanoana (B.L. Rob.) R.M. King \& H. Rob. QRO; 6

Critonia morifolia (Mill.) R.M. King \& H. Rob. QRO; 6, 7, 12

Decachaeta incompta (DC.) R.M. King \& H. Rob. GTO, MICH; 18

Fleischmannia porphyranthema (A. Gray) R.M. King \& H. Rob. GTO, QRO; 12

Fleischmannia pycnocephala (Less.) R.M. King \& H. Rob. GTO, MICH, QRO; 3, 6, 7, 12, 15, 18

Fleischmannia sonorae (A. Gray) R.M. King \&

H. Rob. GTO, MICH; 15, 19

Isocarpha oppositifolia (L.) Cass. QRO; 7

Stevia connata Lag. MICH; 17, 18

Stevia deltoidea Greene GTO, MICH, QRO; 3, 6, 18

Stevia dictyophylla B.L. Rob. GTO, MICH; 3

Stevia elatior Kunth GTO, MICH, QRO; 3, 4, 6, 11, 16, 17, 18, 19, 20

Stevia eupatoria (Spreng.) Willd. GTO, MICH, QRO; 16, 19, 20

Stevia incognita Grashoff MICH, QRO; 16, 20

Stevia jorullensis Kunth GTO, MICH, QRO; 3, 6, $12,16,17,19,20$

Stevia latifolia Benth. GTO, MICH, QRO; 7, 17, 18,19

Stevia lucida Lag. GTO, MICH, QRO; 2, 3, 4, 5 , $6,7,9,11,15,16,17,18,19,20$

Stevia micradenia B.L. Rob. MICH; 17, 18

Stevia micrantha Lag. GTO, MICH, QRO; 3, 6, 8, 11, 12, 13, 15, 17, 18, 19, 20

Stevia monardifolia Kunth GTO, MICH, QRO; 11, 12, 16, 17, 19, 20

Stevia nelsonii B.L. Rob. GTO, MICH, QRO; 18, 19,20

Stevia origanoides Kunth GTO, MICH, QRO; 5, $6,13,17,18,19,20$

Koanophyllon albicaulis (Sch. Bip. ex Klatt) R.M. Stevia ovata Willd. GTO, MICH, QRO; 3, 4, 6, King \& H. Rob. MICH, QRO; 6, 7 18,19

Koanophyllon longifolium (B.L. Rob.) R.M. King \& H. Rob. GTO, QRO; 6, 7

Stevia pilosa Lag. GTO, MICH, QRO; 7, 12, 16, 20

Kyrsteniopsis spinaciifolia (DC.) B.L. Turner GTO, QRO; 5, 6, 7, 12, 16

Microspermum debile Benth. MICH; 18 Mikania cordifolia (L. f.) Willd. MICH, QRO; 7 Mikania micrantha Kunth MICH, QRO; 7

Mikania pyramidata Donn. Sm. QRO; 7

Oxylobus adscendens (Sch. Bip. ex Hemsl.) B.L. Rob. \& Greenm. MICH; 17, 19

Stevia porphyrea $\mathrm{McVaugh}$ GTO, MICH, QRO; 2, 6, 16, 19, 20

Stevia purpusii B.L. Rob. GTO, MICH, QRO; 3

Stevia pyrolifolia Schltdl. QRO; 6

Stevia salicifolia Cav. GTO, MICH, QRO; 3, 5, $11,12,15,18,19,20$

Stevia serrata Cav. GTO, MICH, QRO; 2, 3, 4, 5 , $6,7,10,11,12,13,15,16,17,18,19,20$

Peteravenia schultzii (Schnittsp.) R.M. King \& H. Stevia suaveolens Lag. GTO, MICH, QRO; 3, 20 Rob. GTO, MICH, QRO; 6, 7

Piqueria pilosa Kunth GTO, MICH; 17, 18, 20

Piqueria serrata A. Gray GTO; 5

Piqueria trinervia Cav. GTO, MICH, QRO; 6, 7, $11,12,15,16,17,18,19,20$

Stevia subpubescens Lag. GTO, MICH; 17, 18, 19,20

Stevia tomentosa Kunth GTO, MICH, QRO; 4, $11,12,19,20$

Stevia trifida Lag. GTO, MICH; 18

Stevia aschenborniana Sch. Bip. MICH, QRO; 12 Stevia triflora DC. MICH; 18

Stevia berlandieri A. Gray GTO, QRO; 6, 7, 11

Stevia caracasana DC. MICH, QRO; 16, 18, 19

Stevia viscida Kunth GTO, MICH, QRO; 3, 5, 6, $9,11,15,17,18,19,20$ 
Apéndice. Continuación.

Steviopsis adenosperma (Sch. Bip.) B.L. Turner MICH, QRO; 16

Steviopsis rapunculoides (DC.) R.M. King \& H. Rob. GTO, MICH, QRO; 8, 11

Steviopsis squamulosa (A. Gray) B.L. Turner GTO; 4

Steviopsis thyrsiflora (A. Gray) B.L. Turner GTO, QRO; 3, 5, 12, 15

Tamaulipa azurea (DC.) R.M. King \& H. Rob. QRO; 6, 12

Trichocoronis sessilifolia (Schauer) B. L. Rob. GTO, MICH; 7, 10, 17

\section{Tribu Gnaphalieae}

Chionolaena salicifolia (Bertol.) G.L. Nesom GTO, MICH, QRO; 6, 16, 17, 19, 20

Gamochaeta americana (Mill.) Wedd. GTO, MICH, QRO; 3, 7, 16, 17, 18, 19, 20

Gamochaeta falcata (Lam.) Cabrera GTO, MICH, QRO; 6, 18

Gamochaeta purpurea (L.) Cabrera GTO, QRO; 2, 3

Pseudognaphalium attenuatum (DC.) Anderb. MICH; 17, 18

Pseudognaphalium bourgovii (A. Gray) Anderb. MICH; 13, 17, 19, 20

Pseudognaphalium brachypterum (DC.) Anderb. QRO; 6, 16

Pseudognaphalium canescens (DC.) Anderb. GTO, MICH, QRO; 3, 6, 11, 16, 18

Pseudognaphalium chartaceum (Greenm.) Anderb. GTO, MICH, QRO; 17, 18, 20

Pseudognaphalium conoideum (Kunth) Anderb. MICH; 20

Pseudognaphalium inornatum (DC.) Anderb. GTO, MICH, QRO; 12, 13, 15, 17, 18

Pseudognaphalium liebmannii (Sch. Bip. ex Klatt) Anderb. GTO, MICH, QRO; 20

*Pseudognaphalium luteoalbum (L.) Hilliard \& B.L. Burtt MICH, QRO; 6, 16, 20

Pseudognaphalium oxyphyllum (DC.) Kirp. GTO, MICH, QRO; 12, 17, 20

Pseudognaphalium purpurascens (DC.) Anderb. GTO, MICH, QRO; 10, 16

Pseudognaphalium roseum (Kunth) Anderb. GTO, MICH, QRO; 11, 17, 18, 20
Pseudognaphalium semilanatum (DC.) Anderb. GTO, MICH, QRO; 3, 16, 17, 19, 20

Pseudognaphalium stramineum (Kunth) Anderb. GTO, MICH, QRO; 16, 17

Pseudognaphalium viscosum (Kunth) Anderb. GTO, MICH, QRO; 12, 19, 20

\section{Tribu Gochnatieae}

Gochnatia hypoleuca (DC.) A. Gray GTO, QRO; 6,12

Gochnatia magna M.C. Johnst. GTO, QRO; 5, 6,7

\section{Tribu Helenieae}

Helenium mexicanum Kunth GTO, MICH, QRO; $1,3,4,5,6,8,9,10,11,12,15,16,17,18,19$

Helenium quadridentatum Labill. MICH, QRO; $6,7,11$

Helenium scorzonerifolium (DC.) A. Gray MICH; 19,20

\section{Tribu Heliantheae}

Acmella radicans (Jacq.) R.K. Jansen GTO, $\mathrm{MICH} ; 8,13,14,15,17,18$

Acmella repens (Walter) Rich. GTO, MICH, QRO; 6, 7, 11, 12, 15, 16, 17, 18, 19, 20

Aldama buddleiiformis (DC.) E.E. Schill. \& Panero GTO, MICH, QRO; 3, 9, 10, 15, 16, 17, 18,20

Aldama cornifolia (Kunth) E.E. Schill. \& Panero GTO, MICH, QRO; 3

Aldama dentata La Llave GTO, MICH, QRO; 6, 7, 8, 9, 10, 11, 13, 15, 16, 17, 18, 19, 20

Aldama excelsa (Willd.) E.E. Schill. \& Panero GTO, MICH, QRO; 3, 11, 16, 17, 18 Aldama flava (Hemsl.) E.E. Schill. \& Panero MICH; 18

Aldama ghiesbreghtii (Hemsl.) E.E. Schill. \& Panero MICH, QRO; 11, 16, 17, 18, 20

Aldama hypochlora (S.F. Blake) E.E. Schill. \& Panero GTO, MICH; 17, 18, 19

Aldama linearis (Cav.) E.E. Schill. \& Panero GTO, MICH, QRO; 3, 4, 5, 10, 11, 12, 15, 16, $18,19,20$

Aldama michoacana (B.L. Turner \& F.G. Davies) E.E. Schill. \& Panero MICH; 20 
Apéndice. Continuación.

Aldama pachycephala (DC.) E.E. Schill. \&

Panero GTO, MICH; 4, 9, 17, 18

Aldama palmeri (A. Gray) E.E. Schill. \& Panero

$\mathrm{MICH} ; 13,17$

Aldama subcanescens (S.F. Blake) E.E. Schill. \&

Panero MICH; 18 (endémica de la región)

Ambrosia camphorata (Greene) W.W. Payne

GTO; 4, 9

Ambrosia canescens A. Gray GTO, MICH, QRO; 1, 3, 10, 15, 17, 18

Ambrosia confertiflora DC. GTO, MICH, QRO;

$4,5,6,11$

Ambrosia cordifolia (A. Gray) W.W. Payne GTO, QRO; 4, 5, 6, 11, 12, 16

Ambrosia peruviana Willd. GTO, MICH, QRO;

9, 10, 11, 14, 15, 16, 17, 18, 19, 20

Baltimora geminata (Brandegee) Stuessy QRO; 6

Calanticaria bicolor (S.F. Blake) E.E. Schill. \&

Panero GTO, QRO; 12

Calyptocarpus vialis Less. GTO, MICH, QRO; 5, $6,9,11,17,18$

Chromolepis heterophylla Benth. MICH; 19, 20

Clibadium arboreum Donn. Sm. QRO; 7

Davilanthus huajuapanus (Panero \& Villaseñor)

E.E. Schill. \& Panero MICH; 13

Delilia biflora (L.) Kuntze GTO, MICH, QRO; 6, $9,13,14,17,18,20$

Dendroviguiera pringlei (Fernald) E.E. Schill. \&

Panero GTO, MICH, QRO; 11, 12

Dendroviguiera puruana (Paray) E.E. Schill. \&

Panero MICH; 19

Dendroviguiera quinqueradiata (Cav.) E.E.

Schill. \& Panero GTO, MICH; 2, 9, 10, 15, 17

Dendroviguiera splendens (Panero \& E.E.

Schill.) E.E. Schill. \& Panero GTO, MICH; 8, $13,15,18$

Dugesia mexicana (A. Gray) A. Gray QRO; 16, 20

Eclipta prostrata (L.) L. GTO, MICH, QRO; 6, 7, 8, 13, 14, 15, 16, 17, 18, 19

Euphrosyne partheniifolia DC. GTO, MICH, QRO; 2, 5, 11, 14, 15, 17

Flourensia laurifolia DC. GTO, QRO; 5, 7

Flourensia resinosa (Brandegee) S.F. Blake QRO; 12

Hedosyne ambrosiifolia (A. Gray) Strother GTO, QRO; 12, 16
Helianthella mexicana A. Gray GTO; 1, 5

Helianthus annuus L. GTO, MICH; 10

Helianthus laciniatus A. Gray GTO, MICH,

QRO; 4, 5, 6, 10, 11, 15

Heliomeris multiflora Nutt. GTO, MICH, QRO;

5,12

Heliopsis annua Hemsl. GTO, MICH, QRO; 2, 4, $5,8,9,10,11,13,14,15,16,17,18,20$

Heliopsis buphthalmoides (Jacq.) Dunal GTO, MICH, QRO; 7, 19, 20

Heliopsis longipes (A. Gray) S.F. Blake GTO, QRO; 7

Heliopsis parvifolia A. Gray GTO, QRO; 5, 6, 7

Heliopsis procumbens Hemsl. MICH; 19, 20

Hybridella globosa (Ortega) Cass. GTO, QRO; 4, $5,11,12,16$

Hymenostephium cordatum (Hook. \& Arn.) S.F.

Blake MICH, QRO; 7, 17, 18, 19

Iostephane heterophylla (Cav.) Hemsl. GTO, MICH, QRO; 3, 5, 6, 9, 10, 12, 16, 18, 19, 20

Iostephane madrensis (S. Watson) Strother GTO; 3,4

Jefea lantanifolia (S. Schauer) Strother GTO, QRO; 5, 6, 7, 11, 12, 16

Lagascea angustifolia DC. GTO; 2

Lagascea helianthifolia Kunth MICH, QRO; 6, 7, $12,17,18,19$

Lagascea heteropappus Hemsl. MICH; 17, 18, 19,20

Lasianthaea aurea (D. Don) K.M. Becker GTO, MICH, QRO; 5, 9, 10, 16, 17, 18, 19

Lasianthaea ceanothifolia (Willd.) K.M. Becker GTO, MICH, QRO; 2, 6, 12, 19, 20

Lasianthaea crocea (A. Gray) K.M. Becker GTO, $\mathrm{MICH}$

Lasianthaea fruticosa (L.) K.M. Becker GTO, $\mathrm{MICH}, \mathrm{QRO} ; 7,17,18,19,20$

Lasianthaea palmeri (Greenm.) K.M. Becker MICH, QRO; 16, 17, 18

Melanthera nivea (L.) Small MICH, QRO; 7

Montanoa bipinnatifida (Kunth) K. Koch MICH; $13,17,19$

; Montanoa frutescens (Mairet ex DC.) Hemsl.

GTO, MICH, QRO; 10, 12, 17, 18

Montanoa grandiflora Alamán ex DC. GTO, MICH, QRO; 7, 15, 16, 17, 18, 19, 20 
Apéndice. Continuación.

Montanoa leucantha (Lag.) S.F. Blake GTO,
MICH, QRO; 1, 2, 3, 6, 9, 10, 11, 14, 15, 17, 18,
19, 20
Montanoa mollissima Brongn. ex Groenl. QRO;
6, 11, 12
Montanoa tomentosa Cerv. GTO, MICH, QRO;
4, 5, 6, 7, 10, 11, 12, 13
Otopappus epaleaceus Hemsl. MICH; 19
Parthenium bipinnatifidum (Ortega) Rollins
GTO, MICH, QRO; $2,4,5,9,10,11,12,14,16$,
17, 18

Parthenium confertum A. Gray QRO; 12, 16

Parthenium fruticosum Less. QRO; 7

Parthenium hysterophorus L. GTO, MICH, QRO; $6,7,9,13,14,15,18$

Parthenium incanum Kunth GTO, MICH, QRO; $1,4,5,6,10,12,16$

Perymenium arriagae Rzed. \& Calderón QRO; 6, 12 (endémica de la región)

Perymenium buphthalmoides DC. GTO, MICH, QRO; 3, 5, 9, 11, 15, 16, 18, 19

Perymenium globosum B.L. Rob. MICH; 17

Perymenium mendezii DC. GTO, MICH, QRO; 3, $4,5,10,12,20$

Perymenium moctezumae Rzed. \& Calderón QRO; 12 (endémica de la región)

Perymenium ovalifolium (A. Gray) B.L. Turner QRO; 6, 7, 12

Perymenium sotoarenasii Rzed. \& Calderón QRO; 6 (endémica de la región)

Podachaenium eminens (Lag.) Sch. Bip. MICH, QRO; 7, 18

Rumfordia floribunda DC. MICH; 17

Salmea oligocephala Hemsl. MICH, QRO; 6, 7, 17,18

Salmea scandens (L.) DC. MICH, QRO; 7

Sanvitalia angustifolia Engelm. ex A. Gray GTO, MICH, QRO; 14

Sanvitalia ocymoides DC. GTO, MICH, QRO; 3, $4,10,11,15,18$

Sanvitalia procumbens Lam. GTO, MICH, QRO; 2, 3, 4, 5, 6, 7, 9, 10, 11, 12, 14, 16, 18, 19

Sclerocarpus uniserialis (Hook.) Hemsl. GTO, MICH, QRO; 5, 6, 7, 10, 11, 12, 19

Simsia amplexicaulis (Cav.) Pers. GTO, MICH, QRO; 2, 4, 6, 9, 10, 11, 14, 15, 16, 17, 18, 19, 20
Simsia bicentenarialis Rzed. \& Calderón GTO, QRO; 6, 7, 12 (endémica de la región)

Simsia foetida (Cav.) S.F. Blake GTO, MICH, QRO; 11, 16

Simsia lagasciformis DC. GTO, MICH, QRO; 11, 13,15

Tetrachyron discolor (A. Gray) Wussow \& Urbatsch QRO; 6, 7, 12

Tetrachyron omissum Rzed. \& Calderón QRO; 7 (endémica de la región)

Tetrachyron websteri (Wussow \& Urbatsch) B.L. Turner QRO; 6, 12

Tithonia longiradiata (Bertol.) S.F. Blake QRO; 7

Tithonia rotundifolia (Mill.) S.F. Blake GTO, $\mathrm{MICH}$

Tithonia tubiformis (Jacq.) Cass. GTO, MICH, QRO; 3, 4, 6, 9, 10, 11, 12, 13, 14, 15, 16, 17, 18,20

Verbesina abietifolia Rzed. \& Calderón QRO; 7

Verbesina angustifolia (Benth.) S.F. Blake GTO, MICH; 19

Verbesina breedlovei B.L. Turner MICH; 19, 20

Verbesina carranzae P. Carrillo QRO; 7

Verbesina encelioides (Cav.) A. Gray GTO, MICH, QRO; 4, 5, 6, 7, 10, 11

Verbesina fastigiata B.L. Rob. \& Greenm. MICH; 17, 18, 19, 20

Verbesina grayii (Sch. Bip.) Benth. ex Hemsl. MICH; 17, 18, 19, 20

Verbesina hidalgoana B.L. Turner QRO; 10

Verbesina hypomalaca B.L. Rob. \& Greenm. GTO, MICH; 17, 18, 19

Verbesina klattii B.L. Rob. \& Greenm. MICH; 17, $18,19,20$

Verbesina longipes Hemsl. MICH, QRO; 12

Verbesina mexiae B.L. Turner MICH; 19, 20

Verbesina mollis Kunth GTO, MICH, QRO; 3, 5, $9,11,12,13$

Verbesina montanoifolia B.L. Rob. \& Greenm. GTO, MICH; 13, 17, 18, 19

Verbesina oncophora B.L. Rob. \& Seaton GTO, MICH, QRO; 18, 19, 20

Verbesina pantoptera S.F. Blake MICH; 18

Verbesina parviflora (Kunth) S.F. Blake GTO, MICH, QRO; 3, 17, 18 
Apéndice. Continuación.

Verbesina pedunculosa (DC.) B.L. Rob. GTO, MICH, QRO; 10, 12

Verbesina persicifolia DC. GTO, QRO; 7

Verbesina pietatis McVaugh MICH, QRO; 13, 17,18

Verbesina robinsonii (Klatt) Fernald ex B.L. Rob.

\& Greenm. GTO, QRO; 5, 6, 7, 11, 12

Verbesina seatonii S.F. Blake MICH; 20

Verbesina serrata Cav. GTO, MICH, QRO; 2, 3, $4,5,9,10,11,12,14,15,16,18,19$

Verbesina sphaerocephala A. Gray GTO, $\mathrm{MICH}$; $2,3,8,10,15,17,18$

Verbesina steinmannii P. Carrillo QRO; 7, 11 (endémica de la región)

Verbesina suberosa P. Carrillo GTO; 3 (endémica de la región)

Verbesina tetraptera (Ortega) A. Gray GTO, MICH, QRO; 13, 17, 18, 20

Verbesina turbacensis Kunth MICH, QRO; 7

Verbesina virgata Cav. GTO, MICH, QRO; 4, 5, $6,11,12,15,16,17,18,19,20$

Viguiera dentata (Cav.) Spreng. GTO, MICH, QRO; 4, 5, 6, 7, 8, 9, 10, 11, 12, 13, 15, 17, 18, 19,20

Viguiera paneroana Rzed. \& Calderón QRO; 7 (endémica de la región)

Viguiera sessilifolia DC. GTO, MICH, QRO; 3, 5, 9, 16, 17, 18, 19, 20

Wedelia acapulcensis Kunth GTO, MICH, QRO;

6, 7, 10, 11, 12, 13, 16

Xanthium strumarium L. GTO, MICH, QRO; 4, 9, 10, 11, 12, 13, 14, 15, 16, 18

Zaluzania augusta (Lag.) Sch. Bip. GTO, MICH, QRO; 2, 3, 4, 5, 6, 8, 9, 10, 11, 12, 15, 16

Zaluzania megacephala Sch. Bip. GTO, QRO; 5, $6,11,12$

Zaluzania montagnifolia (Sch. Bip.) Sch. Bip. $\mathrm{MICH} ; 19$

Zaluzania triloba (Ortega) Pers. GTO, QRO; 1, 4, $5,6,10,12,16$

Zinnia americana (Mill.) Olorode \& A.M. Torres GTO, MICH, QRO; 6, 7, 17

Zinnia angustifolia Kunth GTO, MICH, QRO; 3, 9 Zinnia bicolor (DC.) Hemsl. GTO, MICH; 3, 10

Zinnia guanajuatensis (Calderón \& Rzed.) B.L. Turner GTO; 4, 5, 10 (endémica de la región)
Zinnia haageana Regel GTO, MICH, QRO; 8, 10, $11,13,14,15,16,17,18,20$

Zinnia microglossa (DC.) McVaugh GTO; 8

Zinnia peruviana (L.) L. GTO, MICH, QRO; 2, 3, $4,5,6,7,8,9,10,11,12,13,14,15,16,17,18$, 19,20

Zinnia purpusii Brandegee MICH; 17

Zinnia violacea Cav. MICH; 20

Zinnia zamudiana Calderón \& Rzed. QRO; 12 (endémica de la región)

\section{Tribu Inuleae}

Blumea viscosa (Mill.) V.M. Badillo MICH; 20

Pluchea carolinensis (Jacq.) G. Don GTO, MICH, QRO; 5, 6, 7, 10, 12, 13, 19

Pluchea salicifolia (Mill.) S.F. Blake GTO, MICH, QRO; 5, 6, 7, 10, 11, 12, 13, 16, 17, 19, 20

\section{Tribu Liabeae}

Sinclairia glabra (Hemsl.) Rydb. MICH; 17, 19

Sinclairia klattii (B.L. Rob. \& Greenm.) H. Rob. \& Brettell MICH; 18

\section{Tribu Millerieae}

Alloispermum integrifolium (DC.) H. Rob. MICH, QRO; 7, 17, 19

Alloispermum michoacanum (B.L. Rob.) B.L. Turner MICH; 17, 19, 20

Alloispermum palmeri (S. Watson ex A. Gray)

C.F. Fernández \& Urbatsch MICH; 17

Alloispermum scabrum (Lag.) H. Rob. GTO, MICH, QRO; 3, 5, 6, 12, 13, 17, 18, 19, 20

Desmanthodium fruticosum Greenm. MICH; 19

Dyscritothamnus filifolius B.L. Rob. GTO, QRO; 6,12

Dyscritothamnus mirandae Paray GTO, QRO; 5, 6,12

Galinsoga longipes Canne GTO, MICH; 4, 17, 19 Galinsoga parviflora Cav. GTO, MICH, QRO; 4, $5,9,10,11,12,13,14,15,16,17,18,19,20$

Galinsoga triradiata Canne MICH; 17

Guardiola mexicana Humb. \& Bonpl. GTO, MICH; 10, 13, 17, 18, 19, 20

Jaegeria bellidiflora (Sessé \& Moc. ex DC.) A.M. Torres \& Beaman MICH, QRO; 12, 17, 18, 19, 20 
Apéndice. Continuación.

Jaegeria glabra (S. Watson) B.L. Rob. GTO, MICH, QRO; 16, 19, 20

Jaegeria hirta (Lag.) Less. GTO, MICH, QRO; 3, $5,6,7,13,15,16,17,18,19,20$

Jaegeria macrocephala Less. MICH; 17, 18

Jaegeria pedunculata Hook. \& Arn. GTO, MICH; 18, 19

Jaegeria purpurascens B.L. Rob. GTO, QRO; 9, 16

Melampodium americanum L. MICH, QRO; 18

Melampodium aureum Brandegee MICH; 18, 19

Melampodium bibracteatum S. Watson MICH, QRO; 16, 17, 19

Melampodium divaricatum (Rich.) DC. GTO, MICH, QRO; 6, 7, 10, 11, 13, 14, 15, 17, 18, 19, 20

Melampodium glabrum S. Watson GTO, MICH, QRO; 9, 11, 12, 13, 14, 15, 17, 18

Melampodium gracile Less. MICH, QRO; 7, 13, 17

Melampodium longifolium Cerv. ex Cav. GTO, MICH, QRO; 3, 5, 6, 11, 16, 17, 20

Melampodium longipilum B.L. Rob. GTO, MICH, QRO; 5, 6, 12

Melampodium microcephalum Less. GTO, MICH, QRO; 6, 13, 14, 15, 18, 19, 20

Melampodium montanum Benth. GTO, MICH, QRO; 5, 7, 12, 18, 19, 20

Melampodium perfoliatum (Cav.) Kunth GTO, MICH, QRO; 3, 6, 8, 10, 11, 15, 16, 17, 18, 19, 20

Melampodium repens Sessé \& Moc. GTO, MICH, QRO; 11, 16, 17, 19, 20

Melampodium sericeum Lag. GTO, MICH, QRO; 2, 3, 8, 9, 10, 11, 12, 13, 14, 15, 16, 17, 18, 19, 20

Melampodium strigosum Stuessy GTO, MICH, QRO; 5, 6, 11, 12, 16, 17

Milleria quinqueflora $\mathrm{L}$. GTO, MICH, QRO; 6, 7, $8,9,13,14,15,18,19,20$

Oteiza acuminata La Llave GTO, MICH, QRO; $11,16,20$

Sabazia elata (Canne) B.L. Turner QRO; 6 (endémica de la región)

Sabazia glandulosa (Canne) B.L. Turner QRO; 7 (endémica de la región)

Sabazia humilis (Kunth) Cass. MICH; 20
Sabazia multiradiata (Seaton) Longpre GTO, QRO

Schistocarpha bicolor Less. QRO; 7

Schistocarpha eupatorioides (Fenzl) Kuntze QRO; 7

Sigesbeckia agrestis Poepp. \& Endl. GTO, MICH, QRO; 6, 17, 18, 19

Sigesbeckia jorullensis Kunth GTO, MICH, QRO; 16, 17, 19, 20

Smallanthus maculatus (Cav.) H. Rob. GTO, MICH, QRO; 3, 6, 7, 9, 12, 16, 17, 18, 19

Tridax balbisioides (Kunth) A. Gray GTO, $\mathrm{MICH}, \mathrm{QRO} ; 4,11,14,15,16$

Tridax brachylepis Hemsl. MICH; 19

Tridax coronopifolia (Kunth) Hemsl. GTO, MICH, QRO; 3, 4, 5, 6, 9, 10, 11, 12, 13, 14, 15, $17,18,19$

Tridax palmeri A. Gray GTO, QRO; 5, 12

Tridax procumbens $\mathrm{L}$. MICH, QRO; 6

Tridax rosea Sch. Bip. ex B.L. Rob. \& Greenm. GTO, QRO; 5, 11, 12, 16

Tridax trilobata (Cav.) Hemsl. GTO, MICH, QRO; 8, 16, 17, 18, 19, 20

Trigonospermum alexandri Rzed., Calderón \& Pérez-Calix MICH; 18 (endémica de la región)

Trigonospermum annuum McVaugh \& Lask. GTO, MICH, QRO; 2, 3, 6, 13, 15, 17, 18, 19, 20

Trigonospermum melampodioides DC. $\mathrm{MICH}$; 18,19

\section{Tribu Mutisieae}

Chaptalia hololeuca Greene GTO, QRO; 4, 7, 10, 11

Chaptalia lyratifolia Burkart GTO

Chaptalia nutans (L.) Pol. GTO, MICH, QRO; 3, 6, 16

Chaptalia runcinata Kunth MICH; 17, 18, 20

Chaptalia transiliens G.L. Nesom GTO, QRO; 6, 10,12

Leibnitzia lyrata (D. Don) G.L. Nesom GTO, MICH, QRO; 6, 16, 19, 20

Tribu Nassauvieae

Acourtia carranzae L. Cabrera QRO; 6 (endémica de la región)

Acourtia coulteri (A. Gray) Reveal \& R.M. King GTO, QRO; 4, 6, 7 
Apéndice. Continuación.

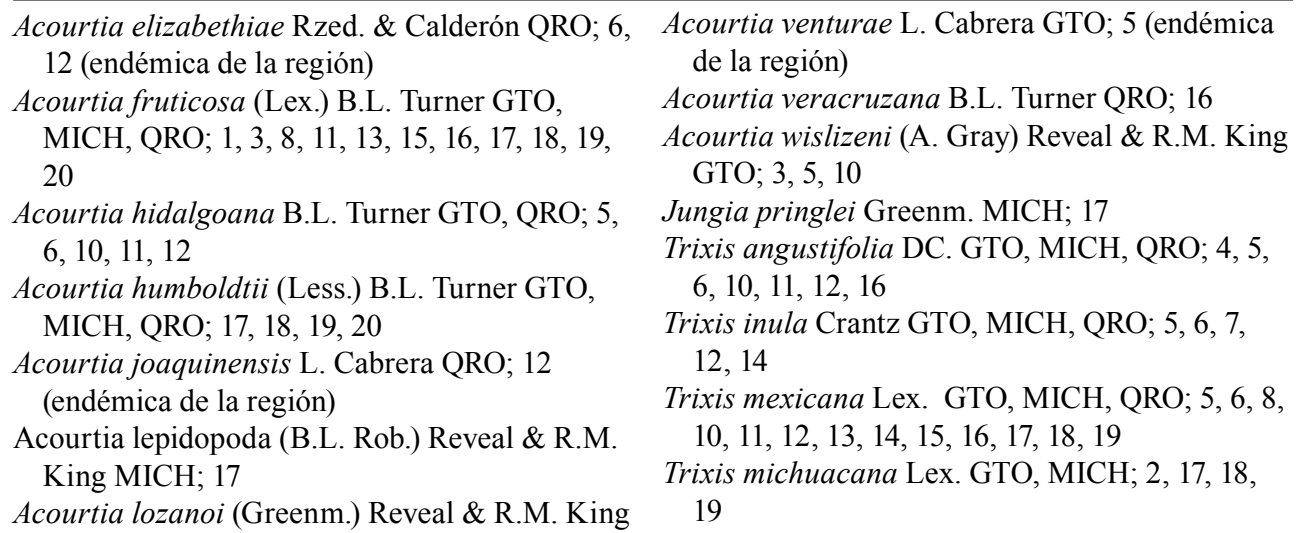

\section{Tribu Neurolaeneae}

Calea ternifolia Kunth GTO, MICH, QRO; 1, 5, $6,7,11,12,18$

Calea urticifolia (Mill.) DC. GTO, MICH, QRO; 7, 18, 19

Neurolaena lobata (L.) Cass. QRO; 7

\section{Tribu Perityleae}

Eutetras palmeri A. Gray GTO; 1, 2, 3

Eutetras pringlei Greenm. GTO, QRO; 4, 11, 12, 15

Galeana pratensis (Kunth) Rydb. GTO, MICH, QRO; 2, 8, 10, 13, 14, 15, 17, 18, 19, 20

Perityle microglossa Benth. GTO, MICH, QRO; 6,7

\section{Tribu Senecioneae}

Barkleyanthus salicifolius (Kunth) H. Rob. \& Brettell GTO, MICH, QRO; 3, 4, 5, 6, 7, 9, 10, 11, 12, 14, 16, 17, 18, 19, 20

Digitacalia jatrophoides (Kunth) Pippen GTO, MICH, QRO; 7, 12, 13, 15, 16, 17, 18, 19, 20

Erechtites hieracifolia (L.) Raf. ex DC. MICH, QRO; 7, 19

Packera bellidifolia (Kunth) W.A. Weber \& A. Löve MICH; 19, 20

Packera coahuilensis (Greenm.) C. Jeffrey QRO; 7, 12

Packera sanguisorbae (DC.) C. Jeffrey GTO, MICH, QRO; 5, 6, 7, 10, 16, 17, 18, 19, 20

Packera tampicana (DC.) C. Jeffrey QRO; 7 
Apéndice. Continuación.

Packera toluccana (DC.) W.A. Weber \& A. Löve GTO, MICH, QRO; 6, 11, 17, 19, 20

Pittocaulon praecox (Cav.) H. Rob. \& Brettell GTO, MICH, QRO; 5, 6, 11, 12, 15, 16, 17, 18, 20

Pittocaulon velatum (Greenm.) H. Rob. \& Brettel MICH; 13, 17, 19, 20

Psacalium amplifolium (DC.) H. Rob. \& Brettell MICH; 19

Psacalium brachycomum (S.F. Blake) H. Rob. \& Brettell MICH; 20 (endémica de la región)

Psacalium cirsiifolium (Zucc.) H. Rob. \& Brettell MICH; 18, 19

Psacalium goldsmithii (B.L. Rob.) H. Rob. \& Brettell MICH; 19

Psacalium holwayanum (B.L. Rob.) Rydb. MICH 19

Psacalium laxiflorum Benth. MICH; 18, 19, 20

Psacalium megaphyllum (B.L. Rob. \& Greenm.) Rydb. GTO, MICH, QRO; 10, 15, 16, 17, 18, 19

Psacalium napellifolium (Schauer) H. Rob. \& Brettell QRO; 12, 16

Psacalium palmeri (Greene) H. Rob. \& Brettell MICH; 17, 18, 20

Psacalium peltatum (Kunth) Cass. GTO, MICH, QRO; 2, 3, 6, 7, 9, 16, 17, 18, 19, 20

Psacalium platylepis (B.L. Rob. \& Seaton) H. Rob. \& Brettell GTO, MICH, QRO; 3, 8, 12, 15,19

Psacalium pringlei (S. Watson) H. Rob. \& Brettell MICH; 20

Psacalium radulifolium (Kunth) $\mathrm{H}$. Rob. \& Brettell GTO, QRO; 2, 3, 5, 6, 7, 9, 10, 11, 12

Psacalium silphiifolium (B.L. Rob. \& Greenm.) H. Rob. \& Brettell MICH; 19

Psacalium sinuatum (Cerv.) H. Rob. \& Brettell GTO, MICH, QRO; 2, 3, 5, 6, 7, 12, 13, 15, 16, $17,18,19$

Psacalium tabulare (Hemsl.) Rydb. MICH; 19

Psacalium tussilaginoides (Kunth) H. Rob. \& Brettell GTO; 5, 9

Pseudogynoxys chenopodioides (Kunth) Cabrera QRO; 7

Roldana albonervia (Greenm.) H. Rob. \& Brettell GTO, MICH, QRO; 15, 17, 18, 19, 20
Roldana angulifolia (DC.) H. Rob. \& Brettell GTO, MICH, QRO; 3, 5, 6, 7, 9, 11, 12, 15, 16, 17, 19, 20

Roldana aschenborniana (Schauer) H. Rob. \& Brettell GTO, QRO; 5, 6, 7, 10, 12, 15, 16

Roldana barba-johannis (DC.) H. Rob. \& Brettell GTO, MICH, QRO; 6, 11, 12, 16, 17, 19, 20

Roldana chapalensis (S. Watson) H. Rob. \& Brettell GTO, MICH; 3, 17, 18, 19

Roldana ehrenbergiana (Klatt) H. Rob. \& Brettell QRO; 6

Roldana guadalajarensis (B.L. Rob.) H. Rob. \& Brettell MICH; 17, 18

Roldana hederifolia (Hemsl.) H. Rob. \& Brettell MICH; 17, 18, 19, 20

Roldana heracleifolia (Hemsl.) H. Rob. \& Brettell GTO, MICH, QRO; 2, 3, 4, 6, 8, 10, 11, 12, 13, $14,15,17,18,20$

Roldana lanicaulis (Greenm.) H. Rob. \& Brettell QRO; 6, 7

Roldana lineolata (DC.) H. Rob. \& Brettell GTO, MICH, QRO; 3, 6, 15, 16, 17, 18, 19, 20

Roldana lobata La Llave GTO, MICH; 15, 17, 18, 19

Roldana mexicana (McVaugh) H. Rob. \& Brettell MICH; 17, 18

Roldana michoacana (B.L. Rob.) H. Rob. \& Brettell GTO, MICH; 16, 17, 18, 19, 20

Roldana platanifolia (Benth.) H. Rob. \& Brettell GTO, MICH; 8, 19

Roldana reticulata (DC.) H. Rob. \& Brettell GTO, MICH, QRO; 3, 11, 12, 16, 17, 19

Roldana sessilifolia (Hook. \& Arn.) H. Rob. \& Brettell GTO, MICH, QRO; 2, 3, 4, 5, 9, 13, 16, 17, 18, 19

Roldana suffulta (Greenm.) H. Rob. \& Brettell GTO, MICH; 15, 19, 20

Senecio alvarezensis Greenm. GTO; 5

Senecio argutus Kunth MICH, QRO; 19

Senecio callosus Sch. Bip. GTO, MICH, QRO; 3, 16, 17, 18, 19, 20

Senecio cinerarioides Kunth GTO, MICH, QRO; $11,19,20$

Senecio deformis Klatt GTO, MICH, QRO; 3, 12, 19

Senecio flaccidus Less. GTO, QRO; 3, 4, 5, 16 
Apéndice. Continuación.

Senecio helodes Benth. MICH; 19, 20

*Senecio inaequidens DC. MICH, QRO; 16, 20

Senecio iodanthus Greenm. GTO, MICH, QRO; 11,17

Senecio multidentatus Sch. Bip. ex Hemsl. GTO, MICH; 5, 6, 15, 17

Senecio picridis S. Schauer GTO, QRO; 5, 6, 7, 9, $11,12,15,16$

Senecio richardsonii B.L. Turner QRO; 7

Senecio stoechadiformis DC. GTO, MICH, QRO; 7, 12, 16, 17, 18, 19, 20

*Senecio vulgaris L. GTO, MICH, QRO; 3, 16, 20

Telanthophora andrieuxii (DC.) H. Rob. \&

Brettell MICH, QRO; 6, 7, 20

Telanthophora grandifolia (Less.) H. Rob. \& Brettell QRO; 7

\section{Tribu Tageteae}

Adenophyllum cancellatum (Cass.) Villarreal GTO, MICH, QRO; 2, 5, 6, 7, 8, 9, 10, 11, 12, $13,14,15,16,17,18,19$

Adenophyllum porophyllum (Cav.) Hemsl. GTO, MICH, QRO; 13, 15, 17, 18, 20

Adenophyllum pulcherrimum (Strother) Villarreal GTO, MICH, QRO; 11, 20

Chrysactinia luzmariae Rzed. \& Calderón GTO; 6 (endémica de la región)

Chrysactinia mexicana A. Gray GTO, MICH, QRO; 4, 5, 12

Chrysactinia pinnata S. Watson QRO; 6, 7

Dyssodia papposa (Vent.) Hitchc. GTO, MICH, QRO; 1, 2, 4, 5, 6, 7, 10, 11, 12, 14, 15, 16, 17, 18, 19, 20

Dyssodia pinnata (Cav.) B.L. Rob. GTO, MICH, QRO; 3, 5, 6, 9, 10, 11, 12, 16, 20

Dyssodia tagetiflora Lag. GTO, MICH, QRO; 2 , 4, 7, 8, 11, 12, 13, 14, 15, 17, 18, 19, 20

Flaveria anomala B.L. Rob. QRO; 6

Flaveria pubescens Rydb. QRO; 6, 12

Flaveria trinervia (Spreng.) C. Mohr GTO,

$\mathrm{MICH}, \mathrm{QRO} ; 4$, 6, 7, 9, 10, 11, 12, 14, 15, 18, 19 Hydropectis stevensii McVaugh GTO; 3

Pectis prostrata Cav. GTO, MICH, QRO; 3, 8, 11, 12, 13, 16, 17, 18, 19

Pectis repens Brandegee GTO, MICH, QRO; 3, 9,11
Porophyllum coloratum (Kunth) DC. GTO, MICH, QRO; 6, 9, 10, 11, 12, 18

Porophyllum linaria (Cav.) DC. GTO, MICH, QRO; 3, 4, 5, 6, 8, 9, 10, 11, 12, 15, 16, 18, 20

Porophyllum macrocephalum DC. GTO, MICH, QRO; 6, 7, 11, 12, 13, 14, 15, 18, 20

Porophyllum viridiflorum (Kunth) DC. GTO, MICH, QRO; 5, 6, 7, 9, 13, 14, 15, 17, 18, 19, 20

Tagetes erecta L. GTO, MICH, QRO; 3, 6, 7, 10, $11,13,16,17,18,19,20$

Tagetes filifolia Lag. GTO, MICH, QRO; 5, 7, 17, 18,19

Tagetes foetidissima DC. GTO, MICH; 17, 18, 19,20

Tagetes jaliscensis Greenm. MICH; 18

Tagetes lucida Cav. GTO, MICH, QRO; 2, 3, 4, 5 , $6,7,8,9,10,12,14,15,16,17,18,19,20$

Tagetes lunulata Ortega GTO, MICH, QRO; 3, 4, $5,6,8,9,10,11,12,14,15,16,17,18,20$

Tagetes micrantha Cav. GTO, MICH, QRO; 1, 3, $4,5,6,11,12,13,15,16,17,18,19,20$

Tagetes moorei $\mathrm{H}$. Rob. QRO; 6, 12

Tagetes persicifolius (Benth.) B.L. Turner GTO, MICH, QRO; 15, 16, 19, 20

Tagetes pringlei $\mathrm{S}$. Watson GTO, MICH, QRO; 8 , $9,16,17,18,19,20$

Tagetes subulata Cerv. GTO, MICH; 8, 9, 15, 16, 17, 18, 19

Tagetes tenuifolia Cav. GTO, MICH, QRO; 6, 11, $12,15,17,18$

Thymophylla acerosa (DC.) Strother GTO; 4, 10

Thymophylla pentachaeta (DC.) Small GTO, QRO; 1, 4, 5, 9, 10, 11, 12, 16

Thymophylla setifolia Lag. GTO, QRO; 3, 4, 5, 6, 9, 10, 12

Thymophylla tenuifolia (Cass.) Rydb. GTO, QRO; 4,10

\section{Tribu Vernonieae}

Bolanosa coulteri A. Gray GTO, MICH; 8

Critoniopsis foliosa (Benth.) H. Rob. GTO, MICH; 10

Critoniopsis heydeana (J.M. Coult.) H. Rob. QRO; 6, 7

Critoniopsis obtusa (Gleason) H. Rob. GTO, QRO; 6, 7, 12 
Apéndice. Continuación.

Critoniopsis salicifolia (DC.) H. Rob. MICH, QRO; 18

Critoniopsis solorzanoana (Rzed. \& Calderón)

$\mathrm{MICH} ; 18$ (endémica de la región)

Critoniopsis tomentosa (Lex.) H. Rob. GTO,

MICH, QRO; 5, 10, 12, 16, 17, 18, 19, 20

Critoniopsis villaregalis (Carvajal) H. Rob.

MICH; 18

Elephantopus mollis Kunth QRO; 7

Leiboldia serrata (D. Don) Gleason QRO; 6, 7

Lepidaploa canescens (Kunth) H. Rob. MICH, QRO; 7

Lepidaploa salzmannii (DC.) H. Rob. QRO; 7

Lepidaploa tortuosa (L.) H. Rob. QRO; 7
Pseudelephantopus spicatus (Aubl.) Rohr MICH, QRO; 6, 7, 17

Vernonanthura cordata (Kunth) H. Rob. MICH; 17,19

Vernonanthura liatroides (DC.) H. Rob. GTO, MICH, QRO; 4, 5, 6, 7, 17, 18, 19, 20

Vernonanthura patens (Kunth) H. Rob. MICH, QRO; 7

Vernonanthura serratuloides (Kunth) H. Rob.

GTO, MICH; 4, 8, 9, 10, 13, 15, 17, 20

Vernonia alamanii DC. GTO, MICH, QRO; 3, 5, $6,7,11,12,13,15,16,17,18,19,20$

Vernonia greggii A. Gray GTO, QRO; 7, 8, 12, 16 